\title{
Quench dynamics in a model with tuneable integrability breaking
}

\author{
F. H. L. Essler, ${ }^{1}$ S. Kehrein, ${ }^{2}$ S. R. Manmana, ${ }^{2}$ and N. J. Robinson ${ }^{1}$ \\ ${ }^{1}$ Rudolf Peierls Centre for Theoretical Physics, Oxford University, Oxford OX1 3NP, United Kingdom \\ ${ }^{2}$ Institut für Theoretische Physik, Georg-August-Universität Göttingen, D-37077 Göttingen, Germany \\ (Received 9 December 2013; revised manuscript received 19 February 2014; published 3 April 2014)
}

\begin{abstract}
We consider quantum quenches in an integrable quantum chain with tuneable-integrability-breaking interactions. In the case where these interactions are weak, we demonstrate that at intermediate times after the quench local observables relax to a prethermalized regime, which can be described by a density matrix that can be viewed as a deformation of a generalized Gibbs ensemble. We present explicit expressions for the approximately conserved charges characterizing this ensemble. We do not find evidence for a crossover from the prethermalized to a thermalized regime on the time scales accessible to us. Increasing the integrability-breaking interactions leads to a behavior that is compatible with eventual thermalization.
\end{abstract}

DOI: 10.1103/PhysRevB.89.165104

PACS number(s): 02.30.Ik, 03.75.Kk, 05.70.Ln

\section{INTRODUCTION}

Important advances in manipulating cold-atomic gases have allowed recent experiments [1-6] to realize essentially unitary time evolution for extended periods of time. Stimulated by such experiments, there has been immense theoretical effort (see, e.g., [7] for a recent review) to understand fundamental questions about the nonequilibrium dynamics of quantum systems: Do observables in a subsystem relax to stationary values? If so, can expectation values be reproduced with a thermal density matrix? What governs how and to which values observables relax?

It is generally accepted that conservation laws and dimensionality play important roles in the time evolution of isolated quantum systems. This is highlighted by the ground-breaking experiments of Kinoshita, Wenger, and Weiss [2]. There, it was found that a three-dimensional condensate of ${ }^{87} \mathrm{Rb}$ atoms driven out of equilibrium rapidly relaxed to a thermal state ("thermalized"), while a condensate constrained to move in a single spatial dimension relaxed slowly to a nonthermal ensemble. It is thought that the presence of additional (approximate) conservation laws in the one-dimensional case lies at the heart of this difference.

Theoretical investigations on translationally invariant models have established two central paradigms for the late-time behavior after a quantum quench: (1) subsystems thermalize and are then described by a Gibbs ensemble (GE) [8]; (2) subsystems do not thermalize, but at late times after the quench are described by generalized Gibbs ensembles (GGEs). There is substantial evidence [9-29] that the latter case applies quite generally to quenches in quantum integrable models, as suggested in a seminal paper by Rigol et al. [30].

The dichotomy in the dependence of stationary behavior after a quench on integrability then poses an intriguing question: what happens if integrability is weakly broken? Does the system thermalize, and if so, how fast does it relax? Might there be an intermediate time scale still governed by the physics of integrability?

Early numerical studies [31] suggested that even with an integrability-breaking term the system does not thermalize on the accessible time scales and system sizes. Studies using analytical methods [32] for $d>1$ and numerical methods in the dynamical mean field limit [33] $(d \rightarrow \infty)$ showed that on intermediate time scales the system approaches a nonthermal quasistationary state (a prethermalization plateau). At later times the system is expected to thermalize [15,34]. Prethermalization plateaus have also been observed in a nonintegrable quantum Ising chain with long-range interactions [35]. It has been suggested recently [36] that the time scale for integrability breaking (leaving the prethermalization plateau) is not necessarily related to the strength of the integrability-breaking term. Experimental evidence for the prethermalization plateau in systems of bosonic cold atoms was reported in Refs. [6,37,38]. In spite of the aforementioned works exhibiting prethermalization plateaus in specific models, a general understanding of if, when, and how such plateaus emerge when integrability is broken remains open. Similarly, a precise characterization of such plateaus in terms of statistical ensembles has not been achieved.

In this work we study the effects of integrability-breaking interactions on the dynamics following a quantum quench. Our setup allows us to compare integrable quantum quenches to quenches where an additional integrability-breaking interaction is added to the postquench Hamiltonian. By combining analytical calculations with time-dependent density matrix renormalization group ( $t-D M R G$ ) results we demonstrate the existence of a prethermalization plateau in the sense that local observables relax to nonthermal values at intermediate times. We characterize this prethermalization plateau in terms of a statistical description that we call the "deformed GGE."

This paper is organized as follows. In Sec. II we introduce the model under study. In Sec. III we consider integrable quenches and compare the observed stationary behavior to thermal and generalized Gibbs ensembles. The continuous unitary transformation technique is introduced and used to study a weakly nonintegrable quench of the model in Sec. IV. In Sec. V we establish the existence of the prethermalized regime and describe the approximately stationary behavior in this regime by constructing a "deformed GGE." The dynamics in the presence of strong integrability-breaking interactions is studied numerically in Sec. VI. Section VII contains a summary and discussion of our main results. Technical details underpinning our analysis are consigned to two appendices. 


\section{THE MODEL}

We consider the following Hamiltonian of spinless fermions with dimerization and density-density interactions:

$$
\begin{aligned}
H(\delta, U)= & -J \sum_{l=1}^{L}\left[1+(-1)^{l} \delta\right]\left(c_{l}^{\dagger} c_{l+1}+\text { H.c. }\right) \\
& +U \sum_{l=1}^{L} c_{l}^{\dagger} c_{l} c_{l+1}^{\dagger} c_{l+1},
\end{aligned}
$$

with periodic boundary conditions. Here $\left\{c_{l}^{\dagger}, c_{j}\right\}=\delta_{l, j}$ and we restrict our attention to the parameter regime $J>0, U \geqslant 0$, and $0<\delta<1$. We work at half filling throughout; i.e., the total number of fermions is $L / 2$. When showing results for the time evolution of observables we measure time in units of $J^{-1}$ throughout. An important characteristic of $H(\delta, U)$ is that fermion number is conserved by virtue of the $U(1)$ symmetry:

$$
c_{j} \longrightarrow e^{i \varphi} c_{j}, \quad \varphi \in[0,2 \pi] .
$$

The presence of the $U(1)$ symmetry is a crucial feature of our model: on the one hand it leads to dramatic simplifications in our analytical calculations, while at the same time it enables us to access very late times in our t-DMRG computations (as compared to existing studies of other nonintegrable onedimensional models).

We note that the Hamiltonian (1) is equivalent to a spin-1/2 Heisenberg $X X Z$ chain with a dimerized $X X$ term as can be shown by means of a Jordan-Wigner transformation. The model with finite $U, \delta$ has previously been studied in order to investigate the effect of interactions on the equilibrium dimerization of the chain $[39,40]$. Density matrix renormalization group calculations suggest that for large values of the interaction parameter $U \gtrsim 4$, the Peierls transition to a dimerized ground state is suppressed [40].

There are several limits in which exact results on the equilibrium phase diagram of $H(\delta, U)$ are available. First, in the absence of interactions $(U=0)$ and for any value of the dimerization parameter $\delta$ we obtain a model of a noninteracting Peierls insulator. Second, for vanishing dimerization $\delta=0$ and $U \geqslant 0$ a Jordan-Wigner transformation maps the model to the spin-1/2 Heisenberg $X X Z$ chain. Finally, in the regime of small $|\delta|$ and $U<J$, the low-energy limit of the model is given by the integrable sine-Gordon model [41].

\section{A. Peierls insulator}

The special case $H(\delta, 0)$ describes a Peierls insulator and can be solved by means of a Bogoliubov transformation

$$
c_{l}=\frac{1}{\sqrt{L}} \sum_{k>0} \sum_{\alpha= \pm} \gamma_{\alpha}(l, k \mid \delta) a_{\alpha}(k) .
$$

Here $a_{\alpha}(k)$ are fermion annihilation operators fulfilling

$$
\left\{a_{\alpha}(k), a_{\beta}(q)\right\}=0, \quad\left\{a_{\alpha}(k), a_{\beta}^{\dagger}(q)\right\}=\delta_{\alpha, \beta} \delta_{k, q} .
$$

The coefficients are chosen as

$$
\gamma_{\alpha}(l, k \mid \delta)=e^{-i k l}\left[u_{\alpha}(k, \delta)+v_{\alpha}(k, \delta)(-1)^{l}\right],
$$

where

$$
\begin{gathered}
v_{\alpha}(k, \delta)=\left[1+\left|\frac{2 J \cos (k)-\epsilon_{\alpha}(k)}{2 \delta J \sin (k)}\right|^{2}\right]^{-1 / 2}, \\
u_{\alpha}(k, \delta)=i v_{\alpha}(k) \frac{2 J \cos (k)-\epsilon_{\alpha}(k)}{2 \delta J \sin (k)}, \\
\epsilon_{\alpha}(k, \delta)=2 \alpha J \sqrt{\delta^{2}+\left(1-\delta^{2}\right) \cos ^{2}(k)} .
\end{gathered}
$$

The "+" and "-" bands are separated by an energy gap of $4 \delta J$. Finally, $\sum_{k>0}$ is a shorthand notation for the momentum sum

$$
\sum_{k>0} f(k)=\sum_{n=1}^{L / 2} f\left(\frac{2 \pi n}{L}\right) .
$$

In terms of the Bogoliubov fermions the Peierls Hamiltonian is diagonal:

$$
H(\delta, 0)=\sum_{k>0} \epsilon_{\alpha}(k, \delta) a_{\alpha}^{\dagger}(k) a_{\alpha}(k) .
$$

\section{B. Integrability-breaking interactions}

Adding interactions to the Peierls Hamiltonian leads to a theory that is not integrable. An exception is the low-energy limit for $|\delta| \ll 1$, which is described by a quantum sine-Gordon model [41]. In the following we will be interested in the regime $0.4 \leqslant \delta \leqslant 0.8$, which is far away from this limit. It is useful to express the density-density interaction in $H(\delta, U)$ in terms of the Bogoliubov fermions diagonalizing $H(\delta, 0)$ :

$$
\begin{aligned}
H_{\mathrm{int}}= & U \sum_{l=1}^{L} c_{l}^{\dagger} c_{l} c_{l+1}^{\dagger} c_{l+1} \\
= & U \sum_{k_{j}>0} V_{\alpha_{1} \alpha_{2} \alpha_{3} \alpha_{4}}\left(k_{1}, k_{2}, k_{3}, k_{4}\right) a_{\alpha_{1}}^{\dagger}\left(k_{1}\right) \\
& \times a_{\alpha_{2}}\left(k_{2}\right) a_{\alpha_{3}}^{\dagger}\left(k_{3}\right) a_{\alpha_{4}}\left(k_{4}\right) \\
V_{\boldsymbol{\alpha}}(\boldsymbol{k})= & \frac{1}{L^{2}} \sum_{l} \gamma_{\alpha_{1}}^{*}\left(l, k_{1} \mid \delta\right) \gamma_{\alpha_{2}}\left(l, k_{2} \mid \delta\right) \gamma_{\alpha_{3}}^{*}\left(l+1, k_{3} \mid \delta\right) \\
& \times \gamma_{\alpha_{4}}\left(l+1, k_{4} \mid \delta\right) \\
= & \frac{1}{L} e^{i\left(k_{3}-k_{4}\right)}\left\{\delta _ { k _ { 1 } + k _ { 3 } , k _ { 2 } + k _ { 4 } } \left[w_{\alpha_{1} \alpha_{2}}\left(k_{1}, k_{2}\right) w_{\alpha_{3} \alpha_{4}}\left(k_{3}, k_{4}\right)\right.\right. \\
& \left.-x_{\alpha_{1} \alpha_{2}}\left(k_{1}, k_{2}\right) x_{\alpha_{3} \alpha_{4}}\left(k_{3}, k_{4}\right)\right] \\
& +\delta_{k_{1}+k_{3}+\pi, k_{2}+k_{4}}\left[x_{\alpha_{1} \alpha_{2}}\left(k_{1}, k_{2}\right) w_{\alpha_{3} \alpha_{4}}\left(k_{3}, k_{4}\right)\right. \\
& \left.\left.-w_{\alpha_{1} \alpha_{2}}\left(k_{1}, k_{2}\right) x_{\alpha_{3} \alpha_{4}}\left(k_{3}, k_{4}\right)\right]\right\}
\end{aligned}
$$

Here we have defined

$$
\begin{gathered}
w_{\alpha \beta}(k, p)=u_{\alpha}^{*}(k, \delta) u_{\beta}(p, \delta)+u \rightarrow v, \\
x_{\alpha \beta}(k, p)=u_{\alpha}^{*}(k, \delta) v_{\beta}(p, \delta)+u \leftrightarrow v .
\end{gathered}
$$

\section{INTEGRABLE QUANTUM QUENCHES}

We first consider a quantum quench of the dimerization parameter $\delta$ in the limit of vanishing interactions $U=0$. The system is initially prepared in the ground state $\left|\Psi_{0}\right\rangle$ of $H\left(\delta_{i}, 0\right)$, 


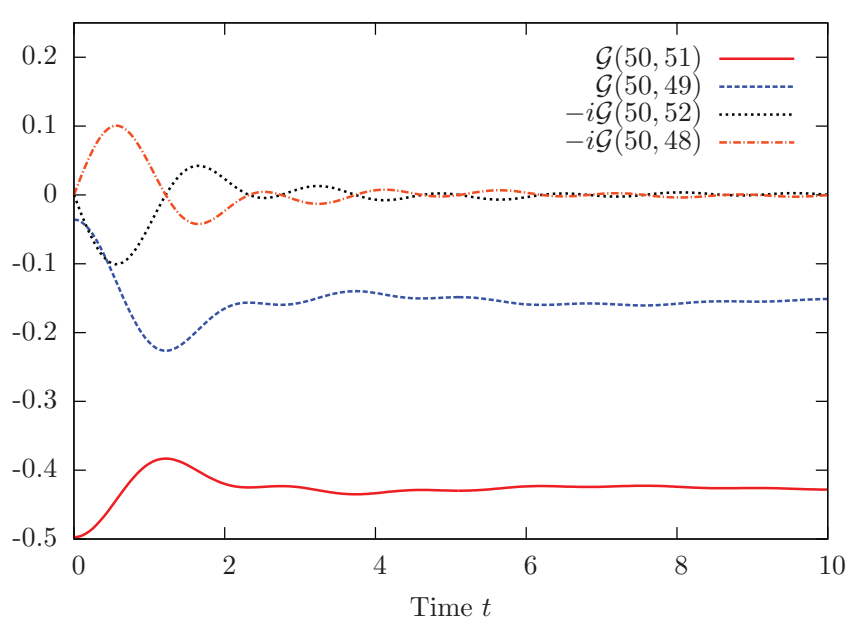

FIG. 1. (Color online) Green's function $G_{0}(j, l, t)$ for a quench with $\delta_{i}=0.75, \delta_{f}=0.25$, and a lattice with $L=100$ sites.

and at time $t=0$ the dimerization is suddenly quenched from $\delta_{i}$ to $\delta_{f}$. At times $t>0$ the system evolves unitarily with the new Hamiltonian $H\left(\delta_{f}, 0\right)$.

The diagonal form of our initial Hamiltonian is

$$
H\left(\delta_{i}, 0\right)=\sum_{\alpha= \pm} \sum_{k>0} \epsilon_{\alpha}\left(k, \delta_{i}\right) b_{\alpha}^{\dagger}(k) b_{\alpha}(k),
$$

and describes two bands of noninteracting fermions. The ground state is obtained by completely filling the "-_" band; i.e.,

$$
\left|\Psi_{0}\right\rangle=\prod_{k} b_{-}^{\dagger}(k)|0\rangle,
$$

where $|0\rangle$ is the fermion vacuum defined by $b_{\alpha}(k)|0\rangle=0$, $\alpha= \pm, k \in(0, \pi]$. At times $t>0$ the system is in the state

$$
\left|\Psi_{0}(t)\right\rangle=e^{-i H\left(\delta_{f}, 0\right) t}\left|\Psi_{0}\right\rangle .
$$

The new Hamiltonian is diagonalized by the Bogoliubov transformation (3)

$$
H\left(\delta_{f}, 0\right)=\sum_{\alpha= \pm} \sum_{k>0} \epsilon_{\alpha}\left(k, \delta_{f}\right) a_{\alpha}^{\dagger}(k) a_{\alpha}(k),
$$

and by virtue of (3) the Bogoliubov fermions $a_{\alpha}(k), a_{\alpha}^{\dagger}(k)$ are linearly related to $b_{\alpha}(k), b_{\alpha}^{\dagger}(k)$. Using this relation it is a straightforward exercise to obtain an explicit expression for the time evolution of the fermion Green's function (see Fig. 1)

$$
\begin{aligned}
G_{0}(j, \ell, t)= & \left\langle\Psi_{0}(t)\left|c_{j}^{\dagger} c_{\ell}\right| \Psi_{0}(t)\right\rangle \\
= & \frac{1}{L} \sum_{k>0} \sum_{\alpha \beta} \gamma_{\alpha}^{*}\left(j, k \mid \delta_{f}\right) \gamma_{\beta}\left(\ell, k \mid \delta_{f}\right) \\
& \times e^{i\left[\epsilon_{\alpha}(k)-\epsilon_{\beta}(k)\right] t} S_{\alpha}^{-}(k) S_{\beta}^{-}(k)^{*}
\end{aligned}
$$

where

$$
S_{\alpha}^{\beta}(k)=u_{\alpha}\left(k, \delta_{f}\right) u_{\beta}^{*}\left(k, \delta_{i}\right)+u \leftrightarrow v .
$$

The late-time behavior can be determined by a stationary phase approximation, which gives

$$
\lim _{t \rightarrow \infty} G_{0}(j, \ell, t) \sim g_{1}(j, \ell)+g_{2}(j, \ell) t^{-3 / 2}+\cdots .
$$

\section{A. Generalized Gibbs ensemble (GGE)}

The stationary state of the dimerization quench is described by a GGE [30]. We now briefly review the construction of the GGE following Refs. $[9,11,12]$. In the thermodynamic limit the system after the quench possesses an infinite number of local conservation laws $I_{a}^{(n)}(a=1,2,3,4, n \in \mathbb{N})$

$$
\left[I_{a}^{(n)}, I_{b}^{(m)}\right]=0, \quad I_{1}^{(1)}=H\left(\delta_{f}, 0\right) .
$$

An explicit construction of these conservation laws is presented in Appendix A. Given these conserved quantities we defined a density matrix

$$
\varrho_{\mathrm{GGE}}=\frac{1}{Z_{\mathrm{GGE}}} \exp \left[-\sum_{a=1}^{4} \sum_{j \geqslant 1} \lambda_{a}^{(j)} I_{a}^{(j)}\right],
$$

where $Z_{\mathrm{GGE}}$ ensures normalization [42]. The Lagrange multipliers are fixed by the requirements that the expectation values of the conserved quantities are the same in the initial state and in the GGE:

$$
\lim _{L \rightarrow \infty} \frac{1}{L}\left\langle\Psi_{0}\left|I_{a}^{(j)}\right| \Psi_{0}\right\rangle=\lim _{L \rightarrow \infty} \frac{1}{L} \operatorname{tr}\left[\varrho_{\mathrm{GGE}} I_{a}^{(j)}\right] .
$$

We then bipartition the system into a segment $\mathrm{B}$ of $\ell$ contiguous sites and its complement $A$ and form the reduced density matrix

$$
\varrho_{\mathrm{GGE}, \mathrm{B}}=\operatorname{tr}_{A}\left[\varrho_{\mathrm{GGE}}\right] .
$$

On the other hand the reduced density matrix of segment B after our quantum quench is simply

$$
\varrho_{B}(t)=\operatorname{tr}_{A}\left[\left|\Psi_{0}(t)\right\rangle\left\langle\Psi_{0}(t)\right|\right] .
$$

At late times after the quench it can be shown by using free fermion techniques (see, e.g., [11]) that

$$
\lim _{t \rightarrow \infty} \lim _{L \rightarrow \infty} \varrho_{B}(t)=\varrho_{\mathrm{GGE}, \mathrm{B}} .
$$

An alternative $[9,14,30]$ but equivalent [12] construction of the GGE is based on the mode occupation numbers

$$
\hat{n}_{\alpha}(k)=a_{\alpha}^{\dagger}(k) a_{\alpha}(k) .
$$

By construction these commute with $H\left(\delta_{f}, 0\right)$ and among themselves, and we can express the density matrix in the form

$$
\varrho_{\mathrm{GGE}}=\frac{1}{Z_{\mathrm{GGE}}} \exp \left[-\sum_{k>0} \sum_{\alpha= \pm} \beta_{k}^{(\alpha)} \hat{n}_{\alpha}(k)\right] .
$$

The Lagrange multipliers are fixed by the conditions

$$
\left\langle\Psi_{0}\left|\hat{n}_{\alpha}(k)\right| \Psi_{0}\right\rangle=\operatorname{tr}\left[\varrho_{\mathrm{GGE}} n_{\alpha}(k)\right],
$$

which are solved by

$$
\begin{aligned}
e^{-\beta_{k}^{(+)}} & =\frac{\left|S_{+}^{-}(k)\right|^{2}}{1-\left|S_{+}^{-}(k)\right|^{2}}, \\
e^{-\beta_{k}^{(-)}} & =\frac{\left|S_{-}^{-}(k)\right|^{2}}{1-\left|S_{-}^{-}(k)\right|^{2}} .
\end{aligned}
$$

Here the functions $S_{\beta}^{\alpha}(k)$ are defined in (18). 


\section{B. GGE vs thermal expectation values}

In the following it will be important to quantify the difference between the GGE constructed above and a Gibbs ensemble (GE)

$$
\varrho_{G}=\frac{1}{Z_{\mathrm{G}}} \exp \left[-\beta_{\mathrm{eff}} H\left(\delta_{f}, 0\right)\right],
$$

constructed by requiring that the average thermal energy density be equal to the energy density in the initial state

$$
\lim _{L \rightarrow \infty} \frac{\left\langle\Psi_{0}\left|H\left(\delta_{f}, 0\right)\right| \Psi_{0}\right\rangle}{L}=\lim _{L \rightarrow \infty} \frac{\operatorname{tr}\left[\varrho_{\mathrm{G}}\left(\beta_{\mathrm{eff}}\right) H\left(\delta_{f}, 0\right)\right]}{L} .
$$

Using the fact that the fermions diagonalizing $H\left(\delta_{f}, 0\right)$ and $H\left(\delta_{i}, 0\right)$ are linearly related by

$$
a_{\alpha}^{\dagger}(k)=S_{\alpha}^{\beta}(k) b_{\beta}^{\dagger}(k),
$$

we can rewrite (31) in the form

$$
\begin{aligned}
& \sum_{k>0} \epsilon_{+}\left(k, \delta_{f}\right)\left[\left|S_{-}^{-}(k)\right|^{2}-\left|S_{+}^{-}(k)\right|^{2}\right] \\
& =\sum_{k>0} \epsilon_{+}\left(k, \delta_{f}\right) \tanh \left[\frac{\beta_{\mathrm{eff}}}{2} \epsilon_{+}\left(k, \delta_{f}\right)\right] .
\end{aligned}
$$

\section{Mode occupation numbers}

In order to exhibit the difference between Gibbs and generalized Gibbs ensembles it is useful to consider the mode occupation numbers, which are given by

$$
\left\langle\hat{n}_{\alpha}(p)\right\rangle= \begin{cases}\frac{1}{1+\exp \left(\beta_{\mathrm{eff}} \epsilon_{\alpha}\left(k, \delta_{f}\right)\right)} & \text { for GE} \\ \frac{1}{1+\exp \left(\beta_{k}^{(\alpha)}\right)} & \text { for GGE. }\end{cases}
$$

Clearly the mode occupation numbers shown in Figs. 2 and 3 are very different in the two ensembles.

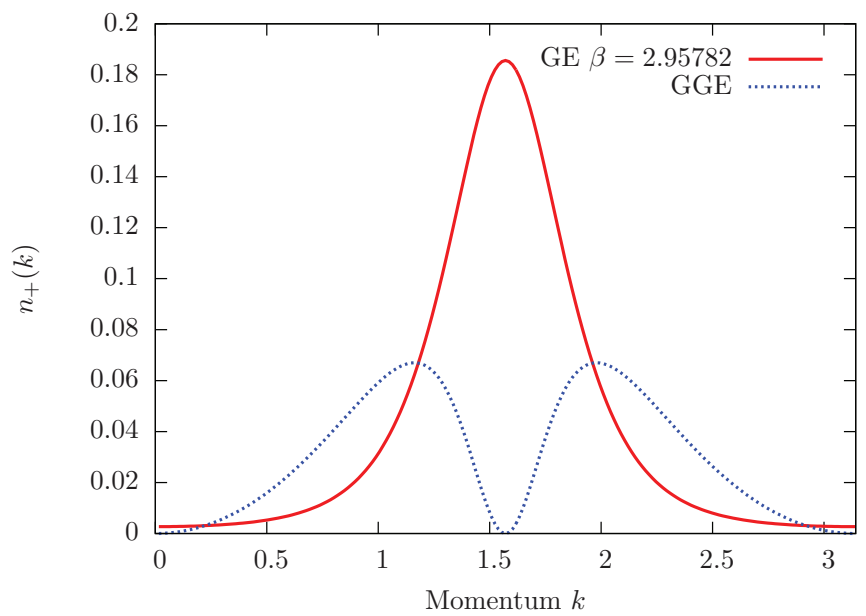

FIG. 2. (Color online) Comparison between the mode occupation numbers $\left\langle n_{+}(k)\right\rangle$ for Gibbs and generalized Gibbs ensembles for a quench with $\delta_{i}=0.75, \delta_{f}=0.25$. The effective inverse temperature for this quench is $\beta_{\text {eff }}=2.95782 \mathrm{~J}$.

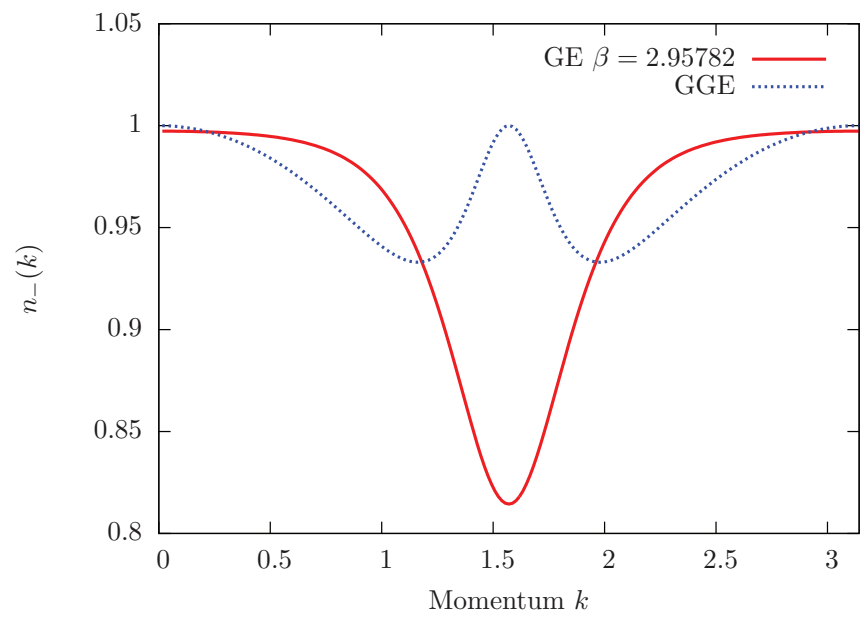

FIG. 3. (Color online) Comparison between the mode occupation numbers $\left\langle n_{-}(k)\right\rangle$ for Gibbs and generalized Gibbs ensembles for a quench with $\delta_{i}=0.75, \delta_{f}=0.25$. The effective inverse temperature for this quench is $\beta_{\text {eff }}=2.95782 \mathrm{~J}$.

\section{Green's function}

As has been emphasized in [11], as we are dealing with the nonequilibrium dynamics of an isolated quantum system, we should focus on the expectation values of local (in space) operators, as descriptions in terms of statistical ensembles most naturally apply to them (see also $[12,43]$ ). We therefore consider the fermionic Green's function in position space, and furthermore focus on its short-distance properties. The Green's functions in the GGE and thermal ensembles are

$$
\left\langle c_{j}^{\dagger} c_{l}\right\rangle=\frac{1}{L} \sum_{p>0} \sum_{\alpha} \gamma_{\alpha}^{*}\left(j, p \mid \delta_{f}\right) \gamma_{\alpha}\left(l, p \mid \delta_{f}\right)\left\langle\hat{n}_{\alpha}(p)\right\rangle,
$$

where the mode occupation numbers are given by (34). In Fig. 4 we show a comparison between the results for the fermion Green's function calculated in the appropriate Gibbs and generalized Gibbs ensembles. We observe that in contrast

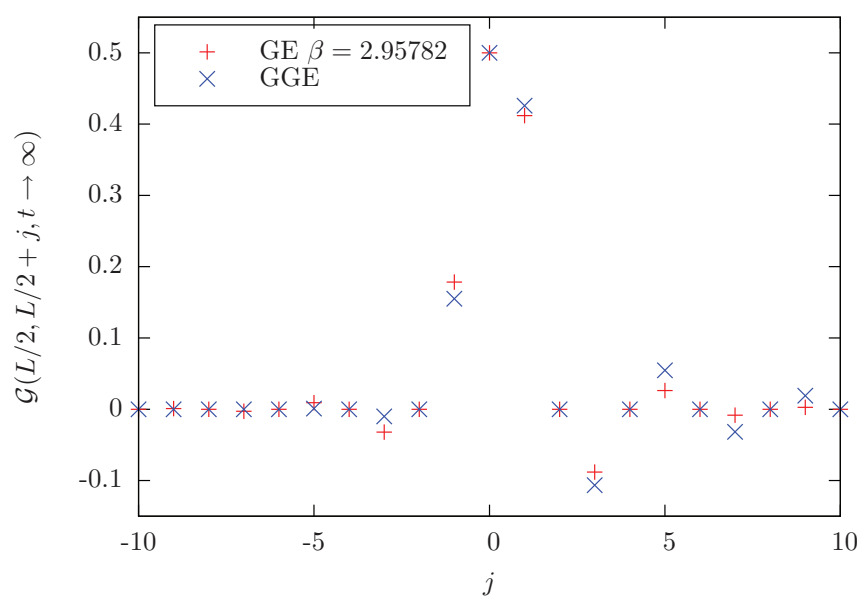

FIG. 4. (Color online) Green's function $\left\langle c_{L / 2}^{\dagger} c_{L / 2+j}\right\rangle$ calculated in the Gibbs and generalized Gibbs ensembles for a quench with $\delta_{i}=0.75, \delta_{f}=0.25$, and a lattice with $L=100$ sites. The effective inverse temperature for this quench is $\beta_{\text {eff }}=2.95782 \mathrm{~J}$. 
to the mode occupation numbers, the difference between the short-distance behavior of the Green's function in the two ensembles is fairly small.

\section{QUENCHING TO A WEAKLY INTERACTING MODEL}

We now modify our quantum quench as follows. We still start out our system in the ground state $\left|\Psi_{0}\right\rangle$ of the pure Peierls Hamiltonian $H\left(\delta_{i}, 0\right)$ given by Eq. (14), but we now quench to $H\left(\delta_{f}, U\right)$, where we consider $U / J$ to be small compared to $\min \left(\delta_{i}, \delta_{f}\right)$. Our main interest is to quantify how a nonzero value of $U$ modifies the dynamics after the quench.

To tackle the quench problem in the nonintegrable weakly interacting model we employ the continuous unitary transformation (CUT) technique [44,45], which has been applied extensively to nonequilibrium problems (see, for example, Refs. [32,46]). We provide a brief overview of the CUT technique for out-of-equilibrium many-body systems and proceed to calculate the time-dependent Green's function and the four-point function.

\section{A. Time-evolution of observables by CUT}

For a nonintegrable interacting model it is no longer possible to calculate the time-evolution induced by the Hamiltonian (1) exactly. We use the CUT technique to obtain a perturbative expansion in $U$ of the time-evolved observables.

The central idea of the CUT method is to construct a sequence of infinitesimal unitary transformations, chosen such that the Hamiltonian becomes successively more energy diagonal. A family of unitarily equivalent Hamiltonians $H(B)$ characterized by the parameter $B$ can be constructed from the solutions of the differential equation

$$
\frac{d H(B)}{d B}=[\eta(B), H(B)],
$$

where $\eta(B)$ is the anti-Hermitian generator of the unitary transformation. Wegner [44] showed that the Hamiltonian in the final basis $H(B=\infty)$ is energy diagonal if $\eta(B)=$ $\left[H_{0}(B), H_{\text {int }}(B)\right]$, where $H_{0}$ is the quadratic part of the Hamiltonian and $H_{\text {int }}$ is the remainder. In practice (36) is used by expanding all operators in power series in an appropriate small parameter, which in our case will be the interaction strength $U$.

Following the transformation with an appropriate choice of generator, the Hamiltonian is energy diagonal (but not integrable). To perform the time evolution we must introduce an additional approximation: We normal-order the interaction term with respect to the initial state $\left|\Psi_{0}\right\rangle$ and neglect the normal-ordered quartic (and higher order) terms:

$$
\begin{aligned}
H(B=\infty) & =H_{0}(B=\infty)+H_{\mathrm{int}}(B=\infty) \\
& =H^{\prime}+: H_{\mathrm{int}}(B=\infty):, \\
\mathcal{U}(t) & \approx \exp \left(-i H^{\prime} t\right),
\end{aligned}
$$

where the time-evolution operator $\mathcal{U}(t)$ depends only on the quadratic Hamiltonian $H^{\prime}$ whose single-particle energies have $\mathcal{O}(U)$ contributions. By construction this approximation introduces a maximal time scale, on which we expect our calculations to be accurate by virtue of the smallness of $U$. Estimating this time scale within the CUT formalism is

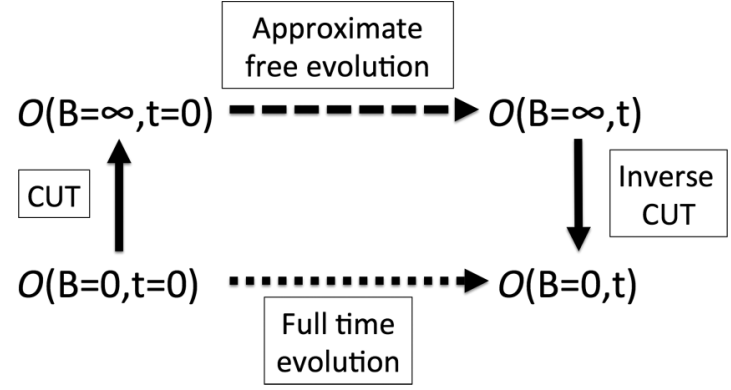

FIG. 5. A schematic of the CUT method for finding the approximate time evolution of the operator $O$ to order $U$.

difficult, as it requires a reliable treatment of the neglected energy diagonal interaction terms. For this reason we extensively compare our CUT results to t-DMRG computations (see Sec. IV E). Importantly, we can perform our CUT calculations for very large systems of hundreds of sites, for which we have verified that finite-size effects do not play a role on time scales less then the revival time (the results shown below are for times less than the revival time). The procedure for calculating the approximate time evolution of observables is shown schematically in Fig. 5.

\section{B. The canonical generator and flow equations for the Hamiltonian}

We start by constructing the "canonical generator" of the unitary transformation [45] given by

$$
\eta(B)=\left[H_{0}(B), H_{\text {int }}(B)\right] .
$$

The flow-dependent operators are defined by

$$
\begin{gathered}
H_{0}(B)=\sum_{\alpha= \pm} \sum_{k>0} \epsilon_{\alpha}(k \mid B) a_{\alpha}^{\dagger}(k) a_{\alpha}(k), \\
H_{\text {int }}(B)=\sum_{k_{j}>0} V_{\boldsymbol{\alpha}}(\boldsymbol{k} \mid B) a_{\alpha_{1}}^{\dagger}\left(k_{1}\right) a_{\alpha_{2}}\left(k_{2}\right) a_{\alpha_{3}}^{\dagger}\left(k_{3}\right) a_{\alpha_{4}}\left(k_{4}\right)+\cdots,
\end{gathered}
$$

where the parameters in the Hamiltonian have been promoted to functions of the flow parameter $B$ and where the dots indicate terms sextic and higher in creation and annihilation operators. The canonical generator is given by

$$
\eta=U \sum_{k_{j}>0} W_{\boldsymbol{\alpha}}(\boldsymbol{k} \mid B) a_{\alpha_{1}}^{\dagger}\left(k_{1}\right) a_{\alpha_{2}}\left(k_{2}\right) a_{\alpha_{3}}^{\dagger}\left(k_{3}\right) a_{\alpha_{4}}\left(k_{4}\right)+\mathcal{O}\left(U^{2}\right),
$$

where

$$
\begin{aligned}
& W_{\boldsymbol{\alpha}}(\boldsymbol{k} \mid B)=V_{\boldsymbol{\alpha}}(\boldsymbol{k} \mid B) E_{\boldsymbol{\alpha}}(\boldsymbol{k} \mid B), \\
& E_{\boldsymbol{\alpha}}(\boldsymbol{k} \mid B)=\epsilon_{\alpha_{1}}\left(k_{1} \mid B\right)-\epsilon_{\alpha_{2}}\left(k_{2} \mid B\right)+\epsilon_{\alpha_{3}}\left(k_{3} \mid B\right)-\epsilon_{\alpha_{4}}\left(k_{4} \mid B\right) .
\end{aligned}
$$

By inserting the canonical generator (40) and the flow Hamiltonian

$$
H(B)=H_{0}(B)+H_{\text {int }}(B)
$$

into the flow equation (36) and integrating the resulting differential equations, we find the flow-dependent 
single-particle energies and interaction vertices:

$$
\begin{gathered}
\epsilon_{\alpha}(k \mid B)=\epsilon_{\alpha}(k \mid B=0), \\
V_{\boldsymbol{\alpha}}(\boldsymbol{k} \mid B)=V_{\boldsymbol{\alpha}}(\boldsymbol{k} \mid B=0) e^{-B E_{\alpha}^{2}(\boldsymbol{k})} .
\end{gathered}
$$

Setting $B=\infty$ we obtain the Hamiltonian in the energydiagonal basis:

$$
\begin{aligned}
H(B=\infty)= & \sum_{\alpha= \pm} \sum_{k>0} \epsilon_{\alpha}(k) a_{\alpha}^{\dagger}(k) a_{\alpha}(k) \\
& +\sum_{k_{j}>0} \breve{V}_{\alpha}(\boldsymbol{k}) a_{\alpha_{1}}^{\dagger}\left(k_{1}\right) a_{\alpha_{2}}\left(k_{2}\right) a_{\alpha_{3}}^{\dagger}\left(k_{3}\right) a_{\alpha_{4}}\left(k_{4}\right) \\
& +\mathcal{O}\left(U^{2}\right)
\end{aligned}
$$

where indeed the interaction vertices conserve energy:

$$
\breve{V}_{\boldsymbol{\alpha}}(\boldsymbol{k}) \equiv V_{\boldsymbol{\alpha}}(\boldsymbol{k} \mid B=\infty)=V_{\boldsymbol{\alpha}}(\boldsymbol{k}) \delta_{E_{\alpha}(\boldsymbol{k}), 0} .
$$

We note that to leading order in $U$ the single-particle energies $\epsilon_{\alpha}(k)$ remain unchanged by the unitary transformation. Having found the energy-diagonal form of the Hamiltonian to leading order we now consider the unitary transformation induced by the canonical generator (40) on the Green's function.

\section{Green's function}

Our main objective is to determine the fermion Green's function on the time-evolved initial state

$$
G(j, l ; t)=\left\langle\Psi_{0}(t)\left|c_{j}^{\dagger} c_{l}\right| \Psi_{0}(t)\right\rangle .
$$

Using the expression for the original fermions in terms of the Bogoliubov fermions $a_{\alpha}(k)$, we see that

$$
\begin{aligned}
c_{j}^{\dagger} c_{l}= & \frac{1}{L} \sum_{k, q>0} \sum_{\alpha, \beta= \pm} \gamma_{\alpha}^{*}\left(j, k \mid \delta_{f}\right) \gamma_{\beta}\left(l, q \mid \delta_{f}\right) \\
& \times \hat{n}_{\alpha \beta}(k, q \mid B=0),
\end{aligned}
$$

where $\gamma_{\alpha}(j, k \mid \delta)$ are defined in Eq. (5) and $\hat{n}_{\alpha \beta}(k, q \mid B=0)=$ $a_{\alpha}^{\dagger}(k) a_{\beta}(q)$. Hence the basic objects we need to calculate are expectation values of $\hat{n}_{\alpha \beta}(p, q \mid B=0)$. This is done by following the procedure set out in Fig. 5. The flow equations

$$
\frac{d \hat{n}_{\alpha \beta}(p, q \mid B)}{d B}=\left[\eta(B), \hat{n}_{\alpha \beta}(p, q \mid B)\right]
$$

are easily constructed to order $\mathcal{O}(U)$ and integrating them gives

$$
\begin{aligned}
\hat{n}_{\alpha \beta}(k, p \mid B)= & \hat{n}_{\alpha \beta}(k, p \mid B=0)+U \sum_{q_{j}>0} N_{\alpha \beta}^{\alpha}(\boldsymbol{q} \mid k, p, B) a_{\alpha_{1}}^{\dagger}\left(q_{1}\right) \\
& \times a_{\alpha_{2}}\left(q_{2}\right) a_{\alpha_{3}}^{\dagger}\left(q_{3}\right) a_{\alpha_{4}}\left(q_{4}\right)+\mathcal{O}\left(U^{2}\right),
\end{aligned}
$$

where we have defined

$$
\begin{aligned}
N_{\alpha \beta}^{\boldsymbol{\alpha}}(\boldsymbol{q} \mid k, p, B)= & \delta_{q_{4}, p} \delta_{\alpha_{4}, \beta} \tilde{V}_{\alpha_{1} \alpha_{2} \alpha_{3} \alpha}\left(q_{1}, q_{2}, q_{3}, k \mid B\right) \\
& +\delta_{q_{2}, p} \delta_{\alpha_{2}, \beta} \tilde{V}_{\alpha_{1} \alpha \alpha_{3} \alpha_{4}}\left(q_{1}, k, q_{3}, q_{4} \mid B\right) \\
& -\delta_{q_{3}, k} \delta_{\alpha_{3}, \alpha} \tilde{V}_{\alpha_{1} \alpha_{2} \beta \alpha_{4}}\left(q_{1}, q_{2}, p, q_{4} \mid B\right) \\
& -\delta_{q_{1}, k} \delta_{\alpha_{1}, \alpha} \tilde{V}_{\beta \alpha_{2} \alpha_{3} \alpha_{4}}\left(p, q_{2}, q_{3}, q_{4} \mid B\right), \\
\tilde{V}_{\boldsymbol{\alpha}}(\boldsymbol{q} \mid B)= & \frac{1-e^{-B\left[E_{\boldsymbol{\alpha}}(\boldsymbol{q})\right]^{2}}}{E_{\boldsymbol{\alpha}}(\boldsymbol{q})} V_{\boldsymbol{\alpha}}(\boldsymbol{q}) .
\end{aligned}
$$

\section{Approximate time evolution}

In the next step of the procedure sketched in Fig. 5 we consider the time evolution induced by the $B=\infty$ Hamiltonian (44). We approximate the time-evolution operator $\mathcal{U}(t)$ by

$$
\mathcal{U}(t)=e^{-i H(B=\infty) t} \approx e^{-i H^{\prime} t},
$$

where the Hamiltonian $H(B=\infty)$ has been replaced by the free fermion Hamiltonian

$$
H^{\prime}=\sum_{\alpha= \pm} \sum_{k>0} \tilde{\epsilon}_{\alpha}(k) a_{\alpha}^{\dagger}(k) a_{\alpha}(k),
$$

with single-particle energies

$$
\tilde{\epsilon}_{\alpha}(k)=\epsilon_{\alpha}(k)+U P_{\alpha}(k) .
$$

The additional term $P_{\alpha}(k)$ is given by

$$
\begin{aligned}
P_{\alpha}(k)= & \sum_{\gamma, \delta} \sum_{q>0}\left[\breve{V}_{\alpha \alpha \gamma \delta}(k, k, q, q)+\breve{V}_{\gamma \delta \alpha \alpha}(q, q, k, k)\right. \\
& \left.-\breve{V}_{\alpha \delta \gamma \alpha}(k, q, q, k)-\breve{V}_{\gamma \alpha \alpha \delta}(q, k, k, q)\right] n_{\gamma \delta}(q),
\end{aligned}
$$

where $\breve{V}_{\boldsymbol{\alpha}}(\boldsymbol{k})$ is defined in Eq. (45). The expectation values $n_{\gamma \delta}(q)=\left\langle\Psi_{0}\left|\hat{n}_{\gamma \delta}(q, q)\right| \Psi_{0}\right\rangle$ taken in the initial state are given by

$$
\begin{aligned}
& n_{--}(k)=\left|S_{-}^{-}(k)\right|^{2}, \\
& n_{++}(k)=\left|S_{+}^{-}(k)\right|^{2}, \\
& n_{+-}(k)=S_{+}^{-}(k) S_{-}^{-}(k)^{*}, \\
& n_{-+}(k)=S_{-}^{-}(k) S_{+}^{-}(k)^{*},
\end{aligned}
$$

where functions $S_{\alpha}^{\beta}(k)$ are defined by Eq. (18). The correction to the single-particle energies $P_{\alpha}(k)$ arises from normalordering the interaction term with respect to the initial state $\left|\Psi_{0}\right\rangle$. The normal-ordering prescription for the quartic term is given by

$$
\begin{aligned}
a_{\alpha_{1}}^{\dagger} a_{\alpha_{2}} a_{\alpha_{3}}^{\dagger} a_{\alpha_{4}}= & : a_{\alpha_{1}}^{\dagger} a_{\alpha_{2}} a_{\alpha_{3}}^{\dagger} a_{\alpha_{4}}:+n_{\alpha_{1} \alpha_{2}}\left(k_{1}\right) \delta_{k_{1}, k_{2}}: a_{\alpha_{3}}^{\dagger} a_{\alpha_{4}}: \\
& +n_{\alpha_{3} \alpha_{4}}\left(k_{3}\right) \delta_{k_{3}, k_{4}}: a_{\alpha_{1}}^{\dagger} a_{\alpha_{2}}: \\
& -n_{\alpha_{1} \alpha_{4}}\left(k_{1}\right) \delta_{k_{1}, k_{4}}: a_{\alpha_{3}}^{\dagger} a_{\alpha_{2}}: \\
& -\left[n_{\alpha_{3} \alpha_{2}}\left(k_{3}\right)-\delta_{\alpha_{2}, \alpha_{3}}\right] \delta_{k_{2}, k_{3}}: a_{\alpha_{1}}^{\dagger} a_{\alpha_{4}}: \\
& +n_{\alpha_{1} \alpha_{2}}\left(k_{1}\right) n_{\alpha_{3} \alpha_{4}}\left(k_{3}\right) \delta_{k_{1}, k_{2}} \delta_{k_{3}, k_{4}}-n_{\alpha_{1} \alpha_{4}}\left(k_{1}\right) \\
& \times\left[n_{\alpha_{3} \alpha_{2}}\left(k_{2}\right)-\delta_{\alpha_{2}, \alpha_{3}}\right] \delta_{k_{1}, k_{4}} \delta_{k_{2}, k_{3}} .
\end{aligned}
$$

The normal-ordered quartic interaction term on the righthand side of (55) has been neglected for the time evolution in Eq. (51). Following this approximation, the time evolution of fermion operators results only in additional phase factors

$$
\mathcal{U}^{\dagger}(t) a_{\alpha}^{\dagger}(k) \mathcal{U}(t)=e^{i \tilde{\epsilon}_{\alpha}(k) t} a_{\alpha}^{\dagger}(k)
$$

Using (56) in (49) provides an explicit expression for the timeevolved operators $\hat{n}_{\alpha \beta}(k, p \mid B=\infty, t)$. In the final step shown in Fig. 5 we reverse the CUT. Integrating back to the initial basis $B=0$, and then taking the expectation value with respect 
to the initial state $\left|\Psi_{0}\right\rangle$, we obtain

$$
\begin{aligned}
\left\langle\hat{n}_{\alpha \beta}(p, q \mid B=0, t)\right\rangle= & \delta_{p, q} e^{i\left(\tilde{\epsilon}_{\alpha}(p)-\tilde{\epsilon}_{\beta}(q)\right) t} n_{\alpha \beta}(p) \\
& +U c_{\alpha \beta}(p, q \mid t)+\mathcal{O}\left(U^{2}\right) .
\end{aligned}
$$

Here the order $U$ piece is

$$
\begin{aligned}
c_{\alpha \beta}(p, q \mid t)= & \sum_{q, r>0} N_{\alpha \beta}^{\gamma}(r, r, q, q|p, q| t) n_{\gamma_{1} \gamma_{2}}(r) n_{\gamma_{3} \gamma_{4}}(q) \\
& -N_{\alpha \beta}^{\gamma}(r, q, q, r|p, q| t) n_{\gamma_{1} \gamma_{4}}(r) \\
& \times\left[n_{\gamma_{3} \gamma_{2}}(q)-\delta_{\gamma_{2}, \gamma_{3}}\right],
\end{aligned}
$$

where we have defined

$$
\begin{aligned}
N_{\alpha \beta}^{\gamma}(\boldsymbol{k}|p, q| t) & =N_{\alpha \beta}^{\gamma}(\boldsymbol{k} \mid p, q, B=\infty)\left[e^{i \tilde{E}_{\gamma}(\boldsymbol{k}) t}-e^{i\left[\tilde{\epsilon}_{\alpha}(p)-\tilde{\epsilon}_{\beta}(q)\right] t}\right], \\
\tilde{E}_{\gamma}(\boldsymbol{k}) & =\tilde{\epsilon}_{\gamma_{1}}\left(k_{1}\right)-\tilde{\epsilon}_{\gamma_{2}}\left(k_{2}\right)+\tilde{\epsilon}_{\gamma_{3}}\left(k_{3}\right)-\tilde{\epsilon}_{\gamma_{4}}\left(k_{4}\right) .
\end{aligned}
$$

Substitution of the observables (57) into Eq. (47) and imposing the momentum-conserving delta functions in the vertices (10) gives the time-dependent Green's function

$$
\begin{aligned}
G(j, l ; t)= & \left\langle\Psi_{0}(t)\left|c_{j}^{\dagger} c_{l}\right| \Psi_{0}(t)\right\rangle \\
= & \frac{1}{L} \sum_{k>0} \sum_{\alpha, \beta= \pm} \gamma_{\alpha}^{*}\left(j, k \mid \delta_{f}\right) \gamma_{\beta}\left(l, k \mid \delta_{f}\right) \\
& \times\left[e^{i\left[\tilde{\epsilon}_{\alpha}(k)-\tilde{\epsilon}_{\beta}(k)\right] t} n_{\alpha \beta}(k)+U c_{\alpha \beta}(k, k \mid t)\right]+\mathcal{O}\left(U^{2}\right) .
\end{aligned}
$$

The remaining momentum sum $\sum_{k>0}$ has to be evaluated numerically.

\section{CUT results for the Green's function}

We first compare the $U \neq 0$ CUT results to the exactly solvable $U=0$ case. Figures 6 and 7 show the nearestneighbor and next-nearest-neighbor Green's functions for the quench $\delta_{i}=0.8 \rightarrow \delta_{f}=0.4$ for several values of $U$.

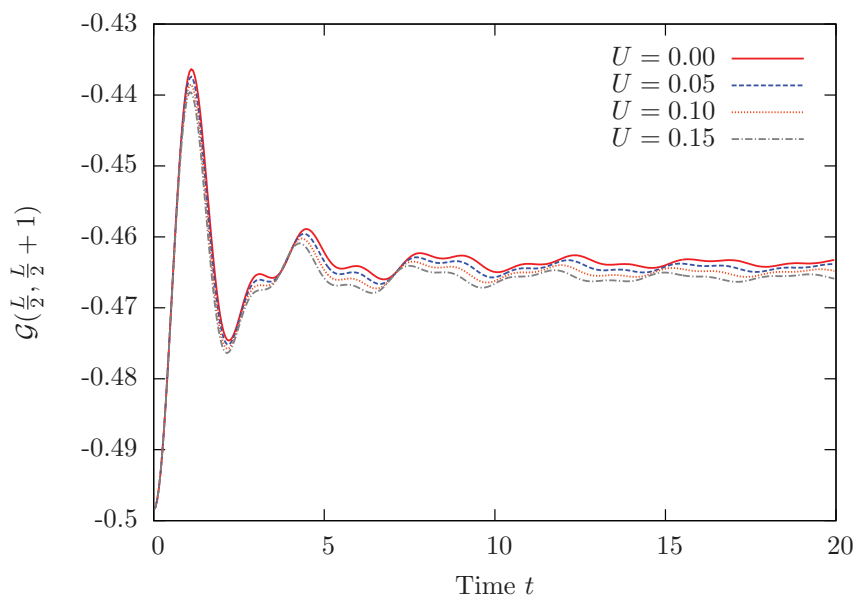

FIG. 6. (Color online) Comparison of exact (solid) $U=0$ nearest-neighbour Green's function $\mathcal{G}(L / 2, L / 2+1)=\left\langle c_{L / 2} c_{L / 2+1}^{\dagger}\right\rangle$ with the CUT results for the quench $\delta_{i}=0.8 \rightarrow \delta=0.4$ and $U_{i}=$ $0 \rightarrow U$ on the $L=100$ chain.

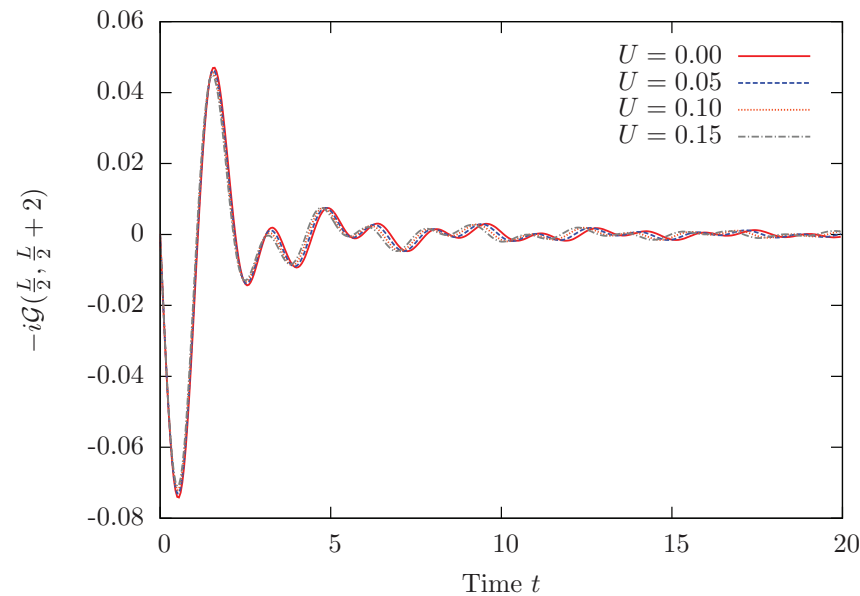

FIG. 7. (Color online) Comparison of exact (solid) $U=0$ nextnearest-neighbor Green's function $\mathcal{G}(L / 2, L / 2+2)=\left\langle c_{L / 2} c_{L / 2+2}^{\dagger}\right\rangle$ with the CUT results for the quench $\delta_{i}=0.8 \rightarrow \delta=0.4$ and $U_{i}=$ $0 \rightarrow U$ on the $L=100$ chain.

With increasing $U$ the periodicity of the oscillations and the asymptotic value of the nearest-neighbor Green's function are continuously deformed away from the noninteracting result. The next-nearest-neighbor Green's function is an imaginary quantity that decays asymptotically to zero for both the noninteracting and CUT result.

In Figs. 8 and 9 we show the fermion Green's function $\mathcal{G}(L / 2, L / 2+j)=\left\langle c_{L / 2} c_{L / 2+j}^{\dagger}\right\rangle$ for separations $j=1,2$ for the quench $\delta_{i}=0.75 \rightarrow \delta=0.5$ and $U_{i}=0 \rightarrow U=0.15$ for the $L=200$ chain. In both cases the long-time decay of the CUT result is compatible with the noninteracting $t^{-3 / 2}$ power-law decay. This is a consequence of the fact that the CUT result (60) has the same general $t$ dependence as the noninteracting case (17).

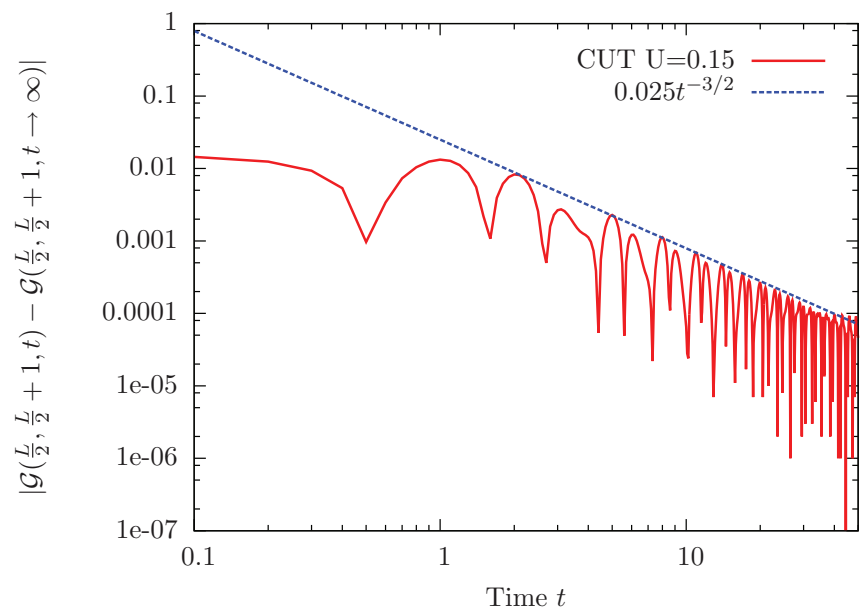

FIG. 8. (Color online) A comparison of the CUT Green's function $|\mathcal{G}(100,101, t)-\mathcal{G}(100,101, t \rightarrow \infty)|$ and the free fermion asymptotic form, Eq. (19), on the $L=200$ chain for the quench $\delta_{i}=0.75 \rightarrow \delta=0.5$ and $U_{i}=0 \rightarrow U=0.15$. The prefactor of the power law $t^{-3 / 2}$ is used as a fit parameter. The revival time of the $L=200$ chain is $t \sim 50$ and the asymptotic value $\mathcal{G}(100,101, t \rightarrow$ $\infty)=-0.482275$. 


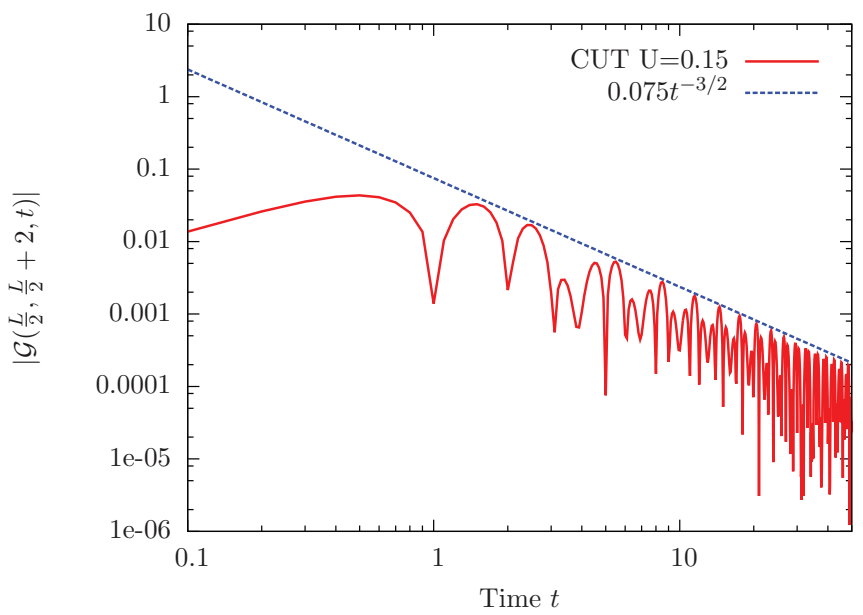

FIG. 9. (Color online) A comparison between the free fermion asymptotic form of the Green's function, Eq. (19), and the CUT result for the quench $\delta_{i}=0.75 \rightarrow \delta=0.5$ and $U_{i}=0 \rightarrow U=0.15$ on the $L=200$ chain. The prefactor of the power law $t^{-3 / 2}$ is used as a fit parameter.

\section{E. Accuracy of the CUT approach: Comparison to time-dependent density matrix renormalization group at small $U / t$}

In order to assess the accuracy of the CUT approach we have carried out extensive comparisons to numerical results obtained by the time-dependent density matrix renormalization group (t-DMRG) algorithm. As is customary in density matrix renormalization group studies, we impose open boundary conditions. We have carried out computations for systems up to $L=200$ lattice sites, but for the purposes of comparing to our CUT results we choose a system size of $L=50$. Up to 1500 density matrix states were kept in the course of the time evolution, and a discarded weight of $\varepsilon=10^{-9}$ was targeted. In order to assess the accuracy of the results at later times, we carried out comparisons to results obtained with a target discarded weight of $\varepsilon=10^{-11}$, and in addition compared to simulations using different time steps of $\delta t=0.005$ or $\delta t=$ 0.01 , respectively. Some details are presented in Appendix B. As shown there, the difference between the results at the end of the time evolution is $\sim 10^{-4}$ or smaller for $L=100$ sites, which means t-DMRG errors are negligible in our comparison to the CUT results.

The revival time $\tau_{r}$ for measurements in the center of a finite chain of noninteracting particles is $L / 2 v_{\max }$, where $L$ is the system size and $v_{\max }$ is the maximal velocity. In the small- $U$ regime of interest here we can obtain a good estimate of $\tau_{r}$ by calculating it in the $U=0$ limit. The estimate can be improved by searching for features associated with revivals at times close to the free fermion estimate. By comparing data with different systems sizes $L$, we have verified that finite-size effects are negligible in the t-DMRG data for times less than the revival time $\tau_{r}$. Finally, we carry out a comparison between CUT and t-DMRG results only for times $t$ sufficiently smaller than $\tau_{r}$. We note that as far as the t-DMRG computations are concerned, we have been able to reach times $\sim 200$ for system size $L=50$. While for short enough times the error in the observable can be estimated as $\sim \sqrt{\varepsilon}$, at longer times, even if the discarded weight

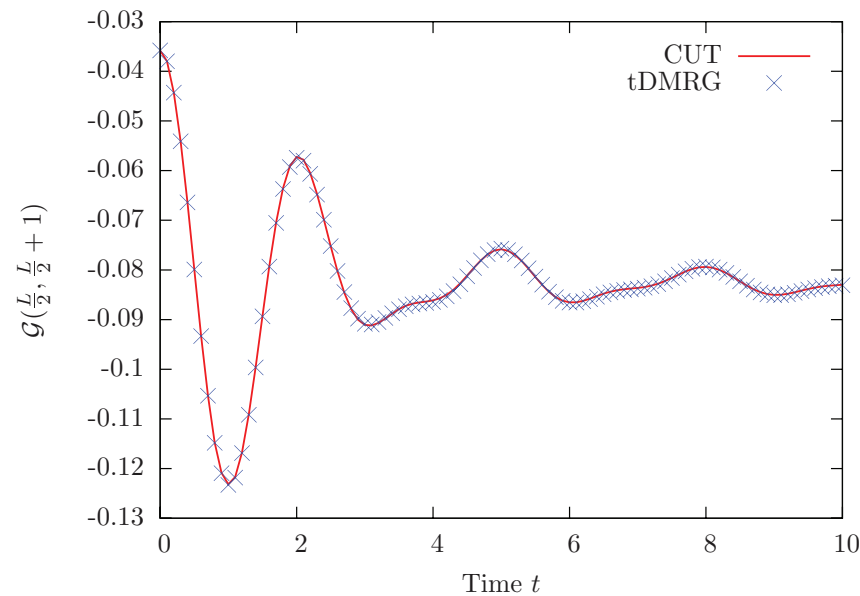

FIG. 10. (Color online) Comparison of the CUT and t-DMRG results for $\mathcal{G}(L / 2, L / 2+1)=\left\langle c_{L / 2} c_{L / 2+1}^{\dagger}\right\rangle$ for the quench $\delta_{i}=$ $0.75 \rightarrow \delta=0.5$ and $U_{i}=0 \rightarrow U=0.15$ on a $L=50$ chain. The revival time for the $L=50$ system is $\tau_{r} \sim 13$.

is kept constant, the accumulation of errors in the course of the sweeps needs to be taken into account. Therefore, for the situations in which times $>20$ are discussed, a more detailed error analysis is necessary, which is presented in Appendix B. In Figs. 10-12 we show a comparison of the CUT and t-DMRG results for the time dependence of the nearestneighbor Green's function $\mathcal{G}(25,26)$ for the length $L=$ 50 chain. We quench the dimerization parameter $\delta_{i}=$ $0.75 \rightarrow \delta=0.5$ and the interaction strength $U=0 \rightarrow U=$ $0.15,0.25,0.5$. There is good, quantitative agreement between the CUT and t-DMRG results provided $U$ is small. The remaining discrepancies have their origin in the order $\mathcal{O}\left(U^{2}\right)$ corrections to the CUT results as is shown in Fig. 13, where we plot the rescaled difference between the t-DMRG data and the CUT result for three values of $U$. The oscillatory nature of these differences can be explained as a "beat frequency"

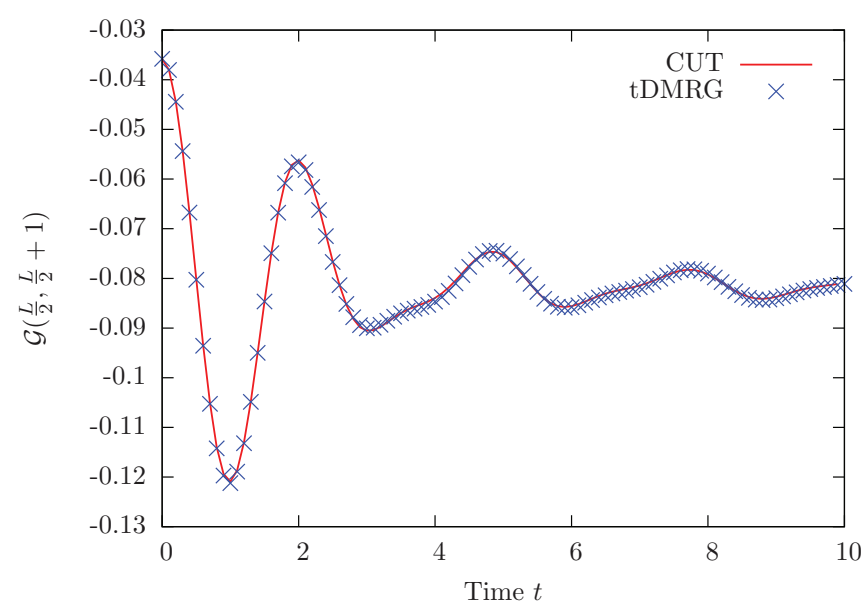

FIG. 11. (Color online) Comparison of the CUT and t-DMRG results for $\mathcal{G}(L / 2, L / 2+1)=\left\langle c_{L / 2} c_{L / 2+1}^{\dagger}\right\rangle$ for the quench $\delta_{i}=$ $0.75 \rightarrow \delta=0.5$ and $U_{i}=0 \rightarrow U=0.25$ on a $L=50$ chain. 


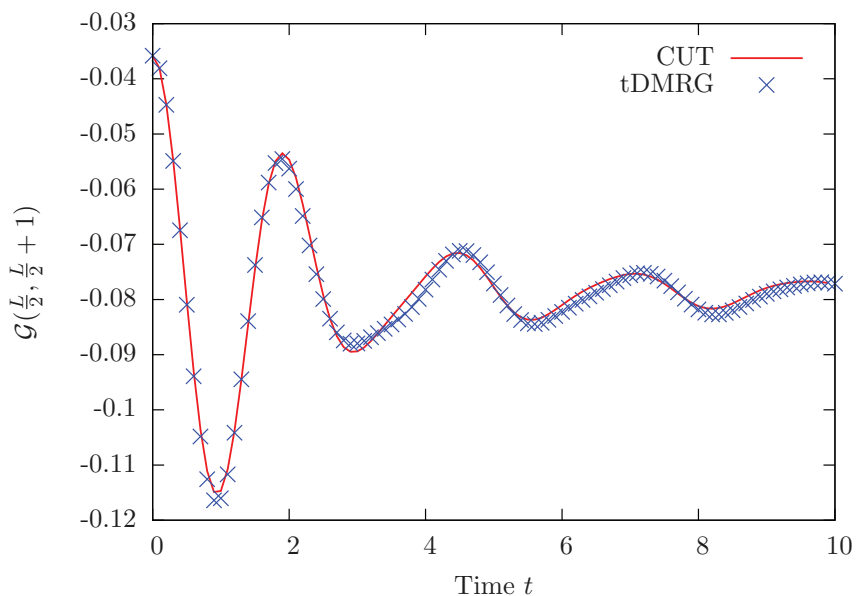

FIG. 12. (Color online) Comparison of the CUT and t-DMRG results for $\mathcal{G}(L / 2, L / 2+1)=\left\langle c_{L / 2} c_{L / 2+1}^{\dagger}\right\rangle$ for the quench $\delta_{i}=$ $0.75 \rightarrow \delta=0.5$ and $U_{i}=0 \rightarrow U=0.5$ on a $L=50$ chain.

arising from subtracting two oscillatory data sets where the frequencies do not match exactly.

Figures 14-16 show that the good agreement between CUTs and t-DMRG is not limited to the nearest-neighbor Green's function by comparing results for $\left\langle\left(c_{L / 2} c_{L / 2+j}^{\dagger}\right)(t)\right\rangle$ with $j=$ 2,3,4 for the case of $U=0.15$.

\section{F. CUT results for the four-point function}

The procedure which we have outlined above for the single-particle Green's function can be generalized to $N$-point functions. The next nonvanishing correlation function is the four-point function

$$
\begin{aligned}
& \left\langle\Psi(t)\left|c_{j}^{\dagger} c_{j^{\prime}} c_{l}^{\dagger} c_{l^{\prime}}\right| \Psi(t)\right\rangle \\
& =\frac{1}{L^{2}} \sum_{q_{j}>0} \sum_{\alpha_{j}= \pm}\left\langle\Psi_{0}\left|\hat{A}_{\alpha}(\boldsymbol{q}, t)\right| \Psi_{0}\right\rangle \\
& \quad \times \gamma_{\alpha_{1}}^{*}\left(j, q_{1}\right) \gamma_{\alpha_{2}}\left(j^{\prime}, q_{2}\right) \gamma_{\alpha_{3}}^{*}\left(l, q_{3}\right) \gamma_{\alpha_{4}}\left(l^{\prime}, q_{4}\right),
\end{aligned}
$$

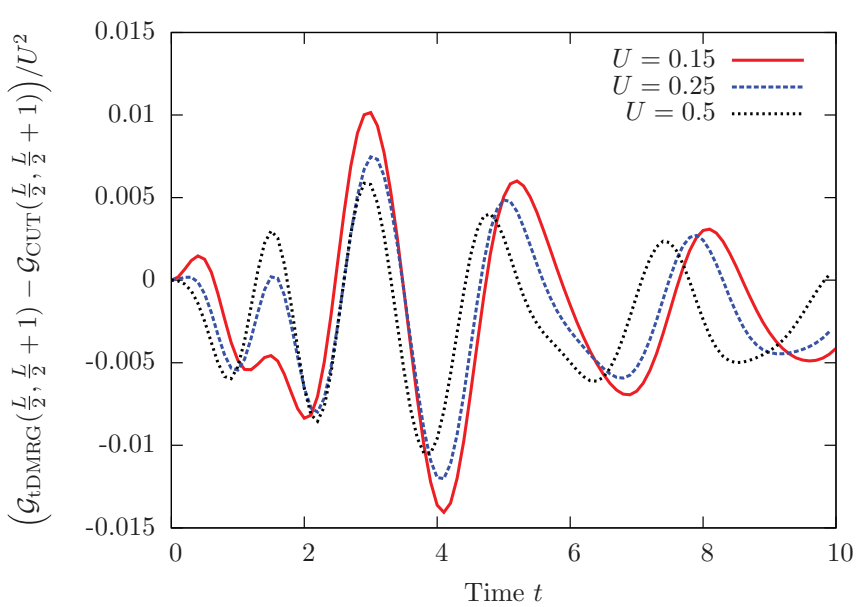

FIG. 13. (Color online) Rescaled difference between the t-DMRG and CUT data for $\mathcal{G}(25,26)$ and different values of $U$.

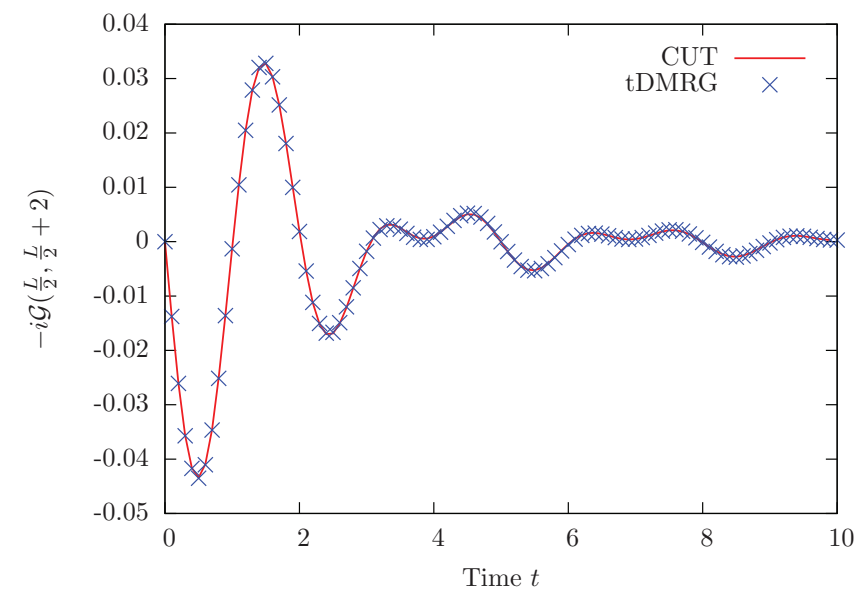

FIG. 14. (Color online) $\mathcal{G}(L / 2, L / 2+2)$ for the quench $\delta_{i}=$ $0.75 \rightarrow \delta=0.5, U_{i}=0 \rightarrow U=0.15$ on an $L=50$ chain.

where $\gamma_{\alpha}(j, k)$ are defined in Eq. (5) and

$$
\hat{A}_{\boldsymbol{\alpha}}(\boldsymbol{q}, t)=a_{\alpha_{1}}^{\dagger}\left(q_{1}, t\right) a_{\alpha_{2}}\left(q_{2}, t\right) a_{\alpha_{3}}^{\dagger}\left(q_{3}, t\right) a_{\alpha_{4}}\left(q_{4}, t\right) .
$$

Going to the $B=\infty$ basis by applying the CUT and then time evolving with (51), we obtain

$$
\begin{aligned}
\hat{A}_{\boldsymbol{\alpha}}(\boldsymbol{q}, t \mid B=\infty) \\
=e^{i \tilde{E}_{\alpha}(\boldsymbol{q}) t} a_{\alpha_{1}}^{\dagger}\left(q_{1}\right) a_{\alpha_{2}}\left(q_{2}\right) a_{\alpha_{3}}^{\dagger}\left(q_{3}\right) a_{\alpha_{4}}\left(q_{4}\right) \\
\quad+U \sum_{k_{j}>0} \sum_{\gamma_{j}= \pm} e^{i\left[\tilde{\sigma}_{\alpha_{1}}\left(q_{1}\right)-\tilde{\epsilon}_{\alpha_{2}}\left(q_{2}\right)\right] t} N_{\alpha_{3} \alpha_{4}}^{\gamma}\left(\boldsymbol{k}\left|q_{3}, q_{4}\right| t\right) \\
\quad \times a_{\alpha_{1}}^{\dagger}\left(q_{1}\right) a_{\alpha_{2}}\left(q_{2}\right) a_{\gamma_{1}}^{\dagger}\left(k_{1}\right) a_{\gamma_{2}}\left(k_{2}\right) a_{\gamma_{3}}^{\dagger}\left(k_{3}\right) a_{\gamma_{4}}\left(k_{4}\right) \\
\quad+U \sum_{k_{j}>0} \sum_{\gamma_{j}= \pm} e^{i\left[\tilde{\sigma}_{\alpha_{3}}\left(q_{3}\right)-\tilde{\epsilon}_{\alpha_{4}}\left(q_{4}\right)\right] t} N_{\alpha_{1} \alpha_{2}}^{\gamma}\left(\boldsymbol{k}\left|q_{1}, q_{2}\right| t\right) a_{\gamma_{1}}^{\dagger}\left(k_{1}\right) \\
\quad \times a_{\gamma_{2}}\left(k_{2}\right) a_{\gamma_{3}}^{\dagger}\left(k_{3}\right) a_{\gamma_{4}}\left(k_{4}\right) a_{\alpha_{3}}^{\dagger}\left(q_{3}\right) a_{\alpha_{4}}\left(q_{4}\right)+\mathcal{O}\left(U^{2}\right), \quad(63)
\end{aligned}
$$

where $\tilde{E}_{\boldsymbol{\alpha}}(\boldsymbol{q})$ and $N_{\alpha \beta}^{\gamma}(\boldsymbol{k}|p, q| t)$ are defined in Eq. (59). Taking the expectation value of Eq. (63) on the initial state using

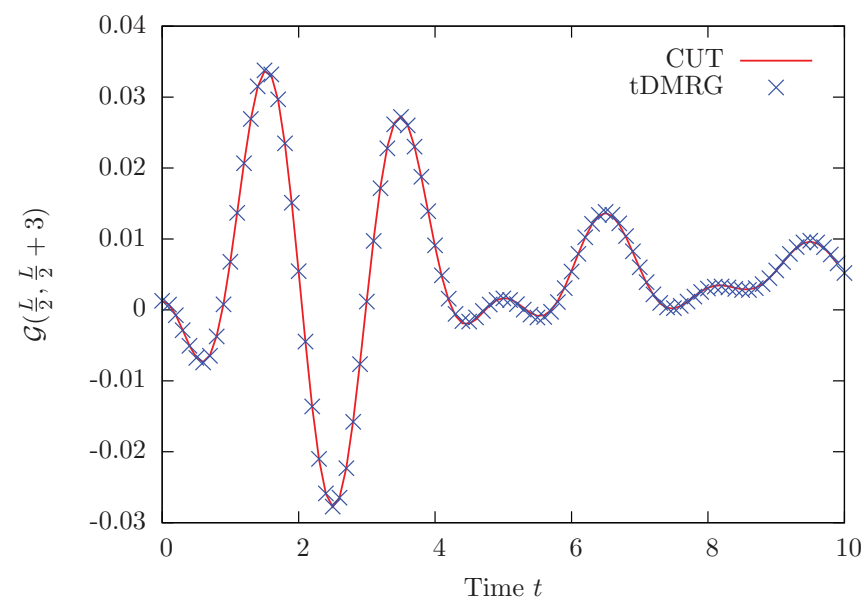

FIG. 15. (Color online) $\mathcal{G}(L / 2, L / 2+3)$ after the quench $\delta_{i}=$ $0.75 \rightarrow \delta=0.5, U_{i}=0 \rightarrow U=0.15$ on an $L=50$ chain. 


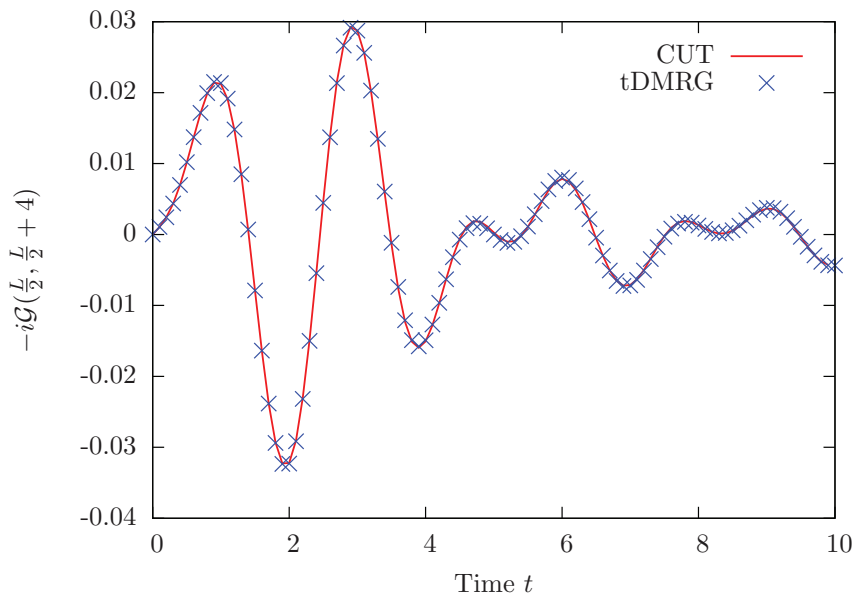

FIG. 16. (Color online) Comparison of the CUT and t-DMRG results for $\mathcal{G}(L / 2, L / 2+4)$ for the quench $\delta_{i}=0.75 \rightarrow \delta=0.5$, $U_{i}=0 \rightarrow U=0.15$ on an $L=50$ chain.

Wick's theorem and substituting into Eq. (61) yields the realspace four-point function.

\section{PRETHERMALIZED REgIME}

The combination of CUT and t-DMRG results establishes that at intermediate times the fermion Green's function $G(j, l, t)$ after a quench $\left(\delta_{i}, 0\right) \rightarrow\left(\delta_{f}, U\right)$ decays in a power-law fashion with approximate exponent $-3 / 2$ to a stationary value; i.e.,

$$
G(j, \ell, t) \longrightarrow g(j, \ell)+\mathcal{O}\left(t^{-3 / 2}\right), \quad J t \lesssim \tau_{0} .
$$

It is very instructive to compare this to the result (19) for the quench $\left(\delta_{i}, 0\right) \rightarrow\left(\delta_{f}, 0\right)$. By virtue of the perturbative nature of the CUT approach, and its excellent agreement with t-DMRG for small $U$ and the time scales relevant to the present discussion, we obtain the following relation between the asymptotic values of the Green's function for the two quenches:

$$
g(j, \ell)-g_{1}(j, \ell)=\mathcal{O}(U) .
$$

We will now show that $g(j, \ell)$ cannot be described by a thermal ensemble, which implies that the stationary regime observed by $\mathrm{t}-\mathrm{DMRG}$ is in fact a prethermalization plateau.

(1) For the quench $\left(\delta_{i}, 0\right) \rightarrow\left(\delta_{f}, 0\right)$ the observed plateau corresponds to the true stationary state and is characterized by a GGE; i.e.,

$$
g_{1}(j, \ell)=\operatorname{tr}\left[\varrho_{\mathrm{GGE}} c_{j}^{\dagger} c_{\ell}\right] .
$$

(2) As we showed in Sec. IIIB, the GGE expectation values for the Green's function are generally different from the thermal expectation values at the appropriate effective inverse temperature $\beta_{0}$ characterizing the quench:

$$
\operatorname{tr}\left[\varrho_{\mathrm{GGE}} c_{j}^{\dagger} c_{\ell}\right]-\operatorname{tr}\left[\varrho_{\mathrm{G}}\left(\beta_{0}\right) c_{j}^{\dagger} c_{\ell}\right]=\mathcal{O}(1)
$$

(3) If the stationary state after the quench $\left(\delta_{i}, 0\right) \rightarrow\left(\delta_{f}, U\right)$ was described by a thermal distribution, its effective inverse temperature $\beta_{\text {eff }}$ would be determined by

$$
\lim _{L \rightarrow \infty} \frac{\left\langle\Psi_{0}\left|H\left(\delta_{f}, U\right)\right| \Psi_{0}\right\rangle}{L}=\lim _{L \rightarrow \infty} \frac{\operatorname{tr}\left[\varrho_{\mathrm{G}}\left(\beta_{\mathrm{eff}}\right) H\left(\delta_{f}, U\right)\right]}{L} .
$$

On the other hand, given that Wick's theorem holds in the state $\left|\Psi_{0}\right\rangle$, we conclude that

$$
\left\langle\Psi_{0}\left|H\left(\delta_{f}, U\right)\right| \Psi_{0}\right\rangle=\left\langle\Psi_{0}\left|H\left(\delta_{f}, 0\right)\right| \Psi_{0}\right\rangle+\mathcal{O}(U) .
$$

Hence

$$
\beta_{\text {eff }}=\beta_{0}+\mathcal{O}(U) .
$$

(4) Combining (70) with (67) we conclude that

$$
\operatorname{tr}\left[\varrho_{\mathrm{GGE}} c_{j}^{\dagger} c_{\ell}\right]-\operatorname{tr}\left[\varrho_{\mathrm{G}}\left(\beta_{\mathrm{eff}}\right) c_{j}^{\dagger} c_{\ell}\right]=\mathcal{O}(1) .
$$

(5) Finally, combining (65), (66), and (71), we conclude that

$$
g(j, \ell)-\operatorname{tr}\left[\varrho_{\mathrm{G}}\left(\beta_{\mathrm{eff}}\right) c_{j}^{\dagger} c_{\ell}\right]=\mathcal{O}(1),
$$

and hence $g(j, \ell)$ is not described by a thermal distribution.

\section{A. Characterization of the prethermalized regime through approximate conservation laws}

In the previous section we have shown that the CUT result cannot produce an effective thermal Gibbs ensemble in the long-time limit. Given that the CUT results for the Green's function are in excellent agreement with t-DMRG data at intermediate times, this establishes the existence of a "prethermalized stationary regime." An obvious question is then how to characterize the statistical ensemble describing the corresponding plateau values of local observables.

\section{Approximate conservation laws}

In our CUT analysis of the nonequilibrium dynamics the generator of time evolution was taken to be

$$
H^{\prime}=\sum_{\alpha= \pm} \sum_{k>0} \tilde{\epsilon}_{\alpha}(k) a_{\alpha}^{\dagger}(k) a_{\alpha}(k)
$$

Clearly the mode occupation number operators $n_{\alpha \alpha}(k)$ commute with $H^{\prime}$, and hence constitute conservation laws (to first order in $U$ ) within our CUT approach. Their pre-images under the CUT, accurate to order $\mathcal{O}(U)$, are simply

$$
\begin{aligned}
\mathcal{Q}_{\alpha}(k)= & a_{\alpha}^{\dagger}(k) a_{\alpha}(k)-U \sum_{q_{j}>0} N_{\alpha \alpha}^{\gamma}(\boldsymbol{q} \mid k, k, B=\infty) \\
& \times a_{\gamma_{1}}^{\dagger}\left(q_{1}\right) a_{\gamma_{2}}\left(q_{2}\right) a_{\gamma_{3}}^{\dagger}\left(q_{3}\right) a_{\gamma_{4}}\left(q_{4}\right) .
\end{aligned}
$$

By construction these operators approximately commute with one another:

$$
\left[\mathcal{Q}_{\alpha}(k), \mathcal{Q}_{\beta}(p)\right]=\mathcal{O}\left(U^{2}\right) .
$$

However, the commutator with the Hamiltonian is in fact

$$
\left[\mathcal{Q}_{\alpha}(k), H\left(\delta_{f}, U\right)\right]=\mathcal{O}(U) ;
$$

i.e., the charges (74) are not (approximately) conserved on an operator level, but only their expectation values with respect to $\left|\Psi_{0}(t)\right\rangle$ are (approximately) time independent. This is a fundamental difference from the proposal put forward 
in Ref. [33] for describing prethermalization plateaus. The charges $\mathcal{Q}_{\alpha}(k)$ have a very transparent physical meaning: they are the number operators for approximately conserved "quasiparticles," and the quartic terms describe the leading contribution to the dressing of the noninteracting fermions.

\section{Approximate description by a "deformed GGE"}

It is natural to attempt a description of the prethermalized regime in terms of a statistical ensemble of the form

$$
\varrho_{\mathrm{PT}}=\frac{1}{Z_{\mathrm{PT}}} \exp \left(\sum_{k, \alpha} \lambda_{k}^{(\alpha)} \mathcal{Q}_{\alpha}(k)\right)
$$

Here the Lagrange multipliers $\lambda_{k}^{(\alpha)}$ are fixed by the requirements

$$
\operatorname{tr}\left[\varrho_{\mathrm{PT}} \mathcal{Q}_{\alpha}(k)\right]=\left\langle\Psi_{0}\left|\mathcal{Q}_{\alpha}(k)\right| \Psi_{0}\right\rangle .
$$

The left-hand side of (78) is most easily evaluated in the $B=$ $\infty$ basis, where it becomes

$$
\frac{1}{Z_{\mathrm{PT}}} \operatorname{tr}\left[e^{\sum_{k, \alpha} \lambda_{k}^{(\alpha)} a_{\alpha}^{\dagger}(k) a_{\alpha}(k)} a_{\alpha}^{\dagger}(k) a_{\alpha}(k)\right]=\frac{1}{1+e^{-\lambda_{k}^{(\alpha)}}} .
$$

The right-hand side of (78) is equal to

$$
\begin{aligned}
& n_{\alpha \alpha}(k)-U \sum_{q_{j}>0} N_{\alpha \alpha}^{\gamma}(\boldsymbol{q} \mid k, B=\infty)\left\{n_{\gamma_{1} \gamma_{2}}\left(q_{1}\right) n_{\gamma_{3} \gamma_{4}}\left(q_{3}\right)\right. \\
& \left.\quad \times \delta_{q_{1}, q_{2}} \delta_{q_{3}, q_{4}}+n_{\gamma_{1} \gamma_{4}}\left(q_{1}\right)\left[\delta_{\gamma_{2}, \gamma_{3}}-n_{\gamma_{3} \gamma_{2}}\left(q_{2}\right)\right] \delta_{q_{1}, q_{4}} \delta_{q_{2}, q_{3}}\right\} .
\end{aligned}
$$

Equating (80) with (79) and using (54) we obtain an explicit expression for the Lagrange multipliers $\lambda_{k}^{(\alpha)}$. The fermion Green's function evaluated with respect to the density matrix (77) is

$$
\begin{aligned}
G_{\mathrm{PT}}(j, \ell)= & \operatorname{tr}\left[\varrho_{\mathrm{PT}} c_{j}^{\dagger} c_{\ell}\right] \\
= & \frac{1}{L} \sum_{q>0} \sum_{\alpha= \pm} \gamma_{\alpha}^{*}\left(j, q \mid \delta_{f}\right) \gamma_{\alpha}\left(\ell, q \mid \delta_{f}\right) \\
& \times \operatorname{tr}\left[\varrho_{\mathrm{PT}} a_{\alpha}^{\dagger}(q) a_{\alpha}(q)\right] .
\end{aligned}
$$

We wish to show that this is equal to the infinite-time limit of the CUT result up to order $\mathcal{O}\left(U^{2}\right)$ corrections; i.e.,

$$
G_{\mathrm{PT}}(j, \ell)=\lim _{t \rightarrow \infty} G(j, \ell ; t)+\mathcal{O}\left(U^{2}\right) .
$$

The trace in (81) is most easily evaluated in the $B=\infty$ basis:

$$
\begin{aligned}
\operatorname{tr}\left[\varrho_{\mathrm{PT}}\right. & \left.a_{\alpha}^{\dagger}(q) a_{\alpha}(q)\right] \\
= & \frac{1}{Z_{\mathrm{PT}}} \operatorname{tr}\left[e^{\sum_{k, \alpha} \lambda_{k}^{(\alpha)} a_{\alpha}^{\dagger}(k) a_{\alpha}(k)} \hat{n}_{\alpha, \alpha}(q, q \mid B=\infty)\right] \\
= & n_{\alpha \alpha}(q)-U \sum_{k_{1,2}>0} N_{\alpha \alpha}^{\gamma}\left(k_{1}, k_{1}, k_{2}, k_{2} \mid q, q, B=\infty\right) \\
& \times n_{\gamma_{1} \gamma_{2}}\left(k_{1}\right) n_{\gamma_{3} \gamma_{4}}\left(k_{2}\right)\left(1-\delta_{\gamma_{1}, \gamma_{2}} \delta_{\gamma_{3}, \gamma_{4}}\right) \\
& -U \sum_{k_{1,2}>0} N_{\alpha \alpha}^{\gamma}\left(k_{1}, k_{2}, k_{2}, k_{1} \mid q, q, B=\infty\right) \\
& \times n_{\gamma_{1} \gamma_{4}}\left(k_{1}\right)\left[\delta_{\gamma_{2}, \gamma_{3}}-n_{\gamma_{3} \gamma_{2}}\left(k_{2}\right)\right]\left(1-\delta_{\gamma_{1}, \gamma_{4}} \delta_{\gamma_{2}, \gamma_{3}}\right) .
\end{aligned}
$$

Substituting (83) into (81) we obtain an expression that indeed agrees with the infinite-time limit of (60) in the thermodynamic limit $L \rightarrow \infty$. This establishes (82). Hence the Green's function $G(j, \ell)$ (for fixed $j, \ell$ in the thermodynamic limit) on the prethermalization plateau is described by the GGE (77) with deformed charges (74). This observation is consistent with a description of local observables on the prethermalization plateau in terms of a deformed GGE. On the other hand there are nonlocal operators, $n_{+-}(k)$ being a simple example, which in fact do not relax at intermediate times and are therefore not described by the ensemble $\varrho_{\mathrm{PT}}$ (without time averaging).

\section{3. "Deformed GGE" description of the four-point function}

The preceding section shows that the value of the Green's function on the prethermalization plateau is given by the deformed GGE $\varrho_{P T}$. We now show that the deformed GGE also reproduces the $t \rightarrow \infty$ expectation value of the CUT result for the four-point function (61). We wish to calculate

$$
\begin{aligned}
\operatorname{tr} & {\left[\varrho_{P T} c_{j}^{\dagger} c_{j^{\prime}} c_{l}^{\dagger} c_{l^{\prime}}\right] } \\
= & \frac{1}{L^{2}} \sum_{q_{j}>0} \sum_{\alpha_{j}= \pm} \gamma_{\alpha_{1}}^{*}\left(j, q_{1}\right) \gamma_{\alpha_{2}}\left(j^{\prime}, q_{2}\right) \gamma_{\alpha_{3}}^{*}\left(l, q_{3}\right) \gamma_{\alpha_{4}}\left(l^{\prime}, q_{4}\right) \\
& \times \operatorname{tr}\left[\varrho_{P T} a_{\alpha_{1}}^{\dagger}\left(q_{1}\right) a_{\alpha_{2}}\left(q_{2}\right) a_{\alpha_{3}}^{\dagger}\left(q_{3}\right) a_{\alpha_{4}}\left(q_{4}\right)\right]
\end{aligned}
$$

with $\varrho_{P T}$ given in (77). As in the previous section, this trace is most easily performed in the $B=\infty$ basis

$$
\begin{aligned}
\operatorname{tr}\left[\varrho_{P T} \hat{A}_{\boldsymbol{\alpha}}(\boldsymbol{q})\right] \\
=\frac{1}{Z_{P T}} \operatorname{tr}\left[e^{\sum_{k, \alpha} \lambda_{k}^{(\alpha)} a_{\alpha}^{\dagger}(k) a_{\alpha}(k)} \hat{A}_{\boldsymbol{\alpha}}(\boldsymbol{q}, B=\infty)\right] \\
=\frac{1}{Z_{P T}} \operatorname{tr}\left[e^{\sum_{k, \alpha} \lambda_{k}^{(\alpha)} a_{\alpha}^{\dagger}(k) a_{\alpha}(k)} \hat{A}_{\boldsymbol{\alpha}}(\boldsymbol{q})\right] \\
\quad+\frac{U}{Z_{P T}} \sum_{k_{j}>0} N_{\alpha_{3} \alpha_{4}}^{\gamma}\left(\boldsymbol{k} \mid q_{3}, q_{4}, \infty\right) \\
\quad \times \operatorname{tr}\left[e^{\sum_{k, \alpha} \lambda_{k}^{(\alpha)} a_{\alpha}^{\dagger}(k) a_{\alpha}(k)} a_{\alpha_{1}}^{\dagger}\left(q_{1}\right) a_{\alpha_{2}}\left(q_{2}\right) \hat{A}_{\boldsymbol{\gamma}}(\boldsymbol{k})\right] \\
\quad+\frac{U}{Z_{P T}} \sum_{k_{j}>0} N_{\alpha_{1} \alpha_{2}}^{\gamma}\left(\boldsymbol{k} \mid q_{1}, q_{2}, \infty\right) \\
\quad \times \operatorname{tr}\left[e^{\sum_{k, \alpha} \lambda_{k}^{(\alpha)} a_{\alpha}^{\dagger}(k) a_{\alpha}(k)} \hat{A}_{\gamma}(\boldsymbol{k}) a_{\alpha_{3}}^{\dagger}\left(q_{3}\right) a_{\alpha_{4}}\left(q_{4}\right)\right]+\mathcal{O}\left(U^{2}\right),
\end{aligned}
$$

where $\hat{A}_{\gamma}(\boldsymbol{k})=a_{\gamma_{1}}^{\dagger}\left(q_{1}\right) a_{\gamma_{2}}\left(q_{2}\right) a_{\gamma_{3}}^{\dagger}\left(q_{3}\right) a_{\gamma_{4}}\left(q_{4}\right)$. The GGE expectation values are easily calculated using Wick's theorem and (78). Retaining only terms up to $\mathcal{O}(U)$ and substituting the result back into (84), we obtain the deformed GGE value for the four-point function on the prethermalization plateau.

In Fig. 17 we plot the difference between the deformed GGE result obtained in this way and the stationary value of the CUT result [found by projecting onto the stationary terms of Eq. (61)] for a number of system sizes and separations. In all cases the difference between the CUT and deformed GGE results scales as $\frac{1}{L}$ and vanishes in the thermodynamic limit $L \rightarrow \infty$. This confirms that the $t \rightarrow \infty$ stationary value of the CUT four-point function is reproduced by the deformed GGE (77). This is a rather nontrivial check of our proposal 


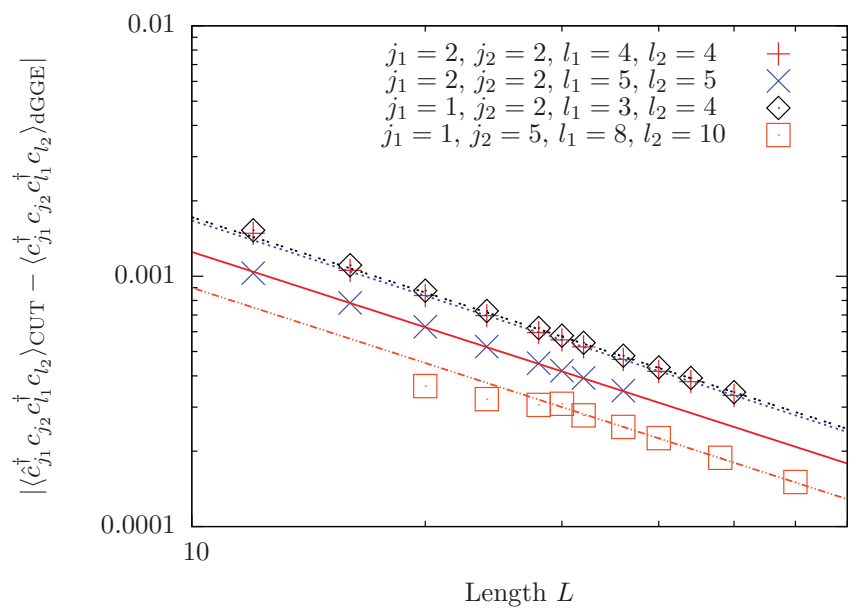

FIG. 17. (Color online) The $L$ dependence of the difference between the deformed GGE and the $t \rightarrow \infty$ CUT result for the four-point function for a number of separations. The solid lines are linear fits $c L^{-1}$ to the data.

that prethermalization plateaus can be described in terms of a deformed GGE.

In Figs. 18 and 19 we present comparisons between t-DMRG results and predictions of the deformed GGE for nearest-neighbor and next-nearest-neighbor density-density correlation functions (84) for the quench $\delta_{i}=0.8 \rightarrow \delta_{f}=$ 0.4 and $U=0 \rightarrow 0.4$. Taking into account that $U_{f}$ is not particularly small, the observed agreement between the two results is quite satisfactory. This supports our assertion that the deformed GGE provides a good description of higher order correlation functions on the prethermalization plateau. We see similarly good agreement for all separations (up to 4 sites) that we explicitly checked. The deformed GGE predictions and the CUT result of Fig. 18 are calculated for system sizes $L=40,50$ rather than $L=100$, because the computational cost of carrying out the momentum sums in the expression for

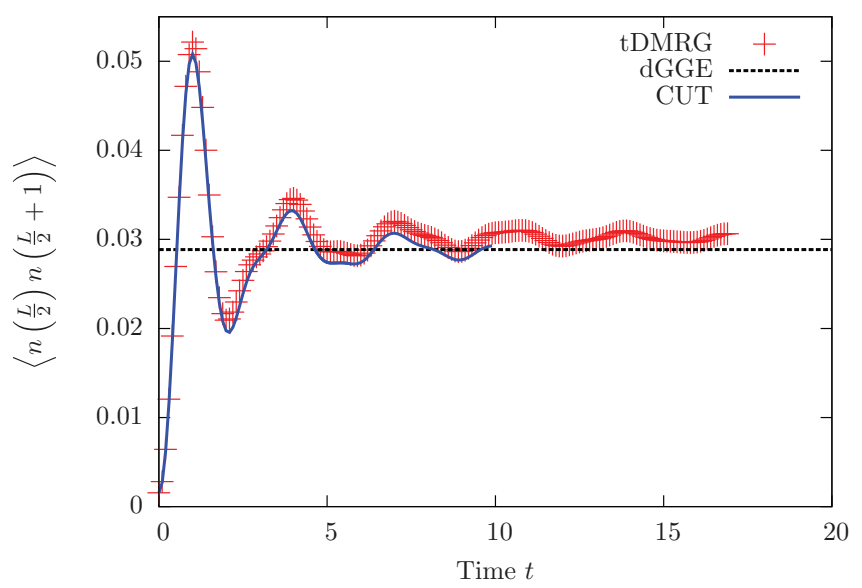

FIG. 18. (Color online) Nearest neighbor density-density correlation function $\left\langle n\left(\frac{L}{2}\right) n\left(\frac{L}{2}+1\right)\right\rangle$ for a quench from $\delta_{i}=0.8 \rightarrow \delta_{f}=$ 0.4 and $U=0 \rightarrow 0.4$ computed by t-DMRG for system size $L=$ 100. For comparison we show CUT results for $L=40$ and the asymptotic value predicted by the $L=50$ deformed GGE.

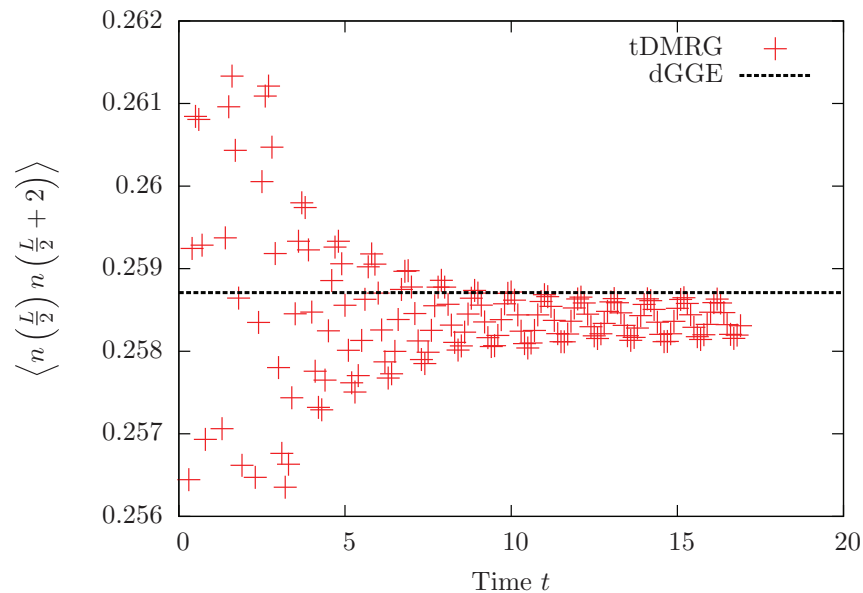

FIG. 19. (Color online) Next-nearest-neighbor density-density correlation function $\left\langle n\left(\frac{L}{2}\right) n\left(\frac{L}{2}+2\right)\right\rangle$ for a quench from $\delta_{i}=0.8 \rightarrow$ $\delta_{f}=0.4$ and $U=0 \rightarrow 0.4$ computed by t-DMRG for system size $L=100$. The correlator relaxes to a stationary value consistent with the deformed GGE prediction (evaluated for $L=50$ ).

the four-point function (61) increases very rapidly with system size.

\section{T-DMRG RESULTS FOR LARGER VALUES OF $U$ AND ABSENCE OF THERMALIZATION ON ACCESSIBLE TIMES SCALE}

In this section we turn to numerical results obtained for quenches to final Hamiltonians with both weak and strong interactions, i.e., when $U \gtrsim\left|\delta_{i}-\delta_{f}\right|$. As can be seen, in all cases the time evolution seems to reach a plateau and remains - on the accessible time scales-on this plateau. This is observed for quenches starting from a noninteracting initial state as well as when $U_{\text {ini }}=5$.

\section{A. Extent of prethermalization plateaus}

The first issue we want to address is the time scale over which we observe prethermalization plateaus. In Figs. 10-12 and 14-16 results are shown only up to $t \approx 10$ in order to avoid revivals. The prethermalization plateau for $U=0.4$ persists to much later times of at least $t \approx 30$, as can be seen in Fig. 20, where we present data for $L=16, L=100, L=200$. On the accessible time scales there is no sign that the $L=200$ system starts to deviate from the plateau at late times.

\section{B. Time averages}

A standard method for extracting stationary values of observables from finite systems is to consider time-averaged quantities; e.g.,

$$
\frac{1}{T} \int_{0}^{T} d t G(L / 2, L / 2+1) .
$$

For the $L=16$ system shown in Fig. 20 the average over long times is in good agreement with the plateau value for the $L=100$ and $L=200$ data. One question that can be asked is whether time averages may reveal signs of the system deviating from the prethermalization plateau. In order to investigate this 


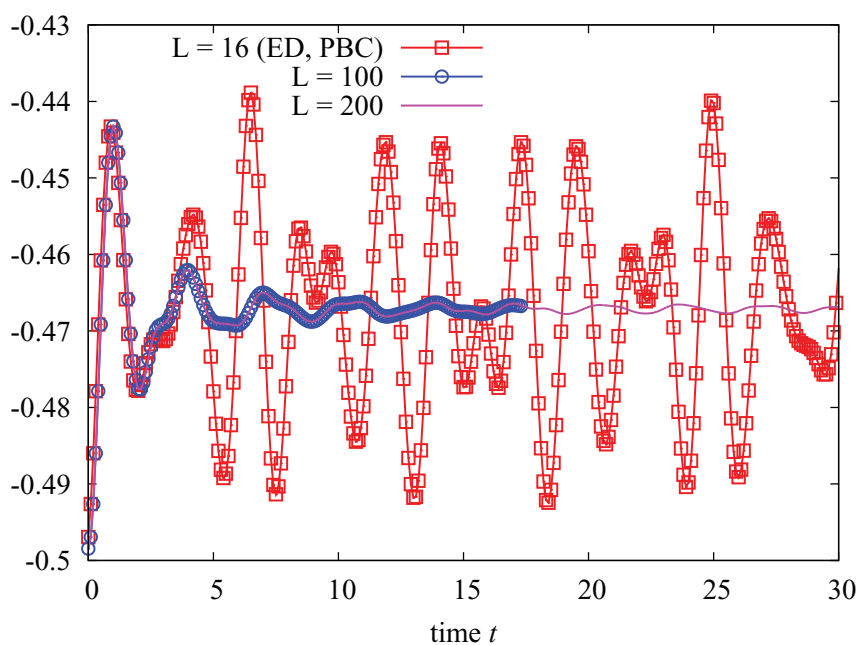

FIG. 20. (Color online) Time evolution of $\mathcal{G}(L / 2, L / 2+1)$ for quenches with $\delta_{i}=0.8 \rightarrow \delta_{f}=0.4$ and $U_{i}=0 \rightarrow U=0.4$ and system sizes $L=16, L=100$, and $L=200$ sites. The data for $L=16$ are ED results for systems with periodic boundary conditions (PBCs) and are seen to exhibit many revivals.

issue, we have carried out t-DMRG simulations for a $L=50$ system up to very late times $t=200$. The results are shown in Fig. 21. Time averages of the t-DMRG data do not reveal any signs of deviations from the plateau value at late times.

\section{The role of interactions in the prequench and postquench Hamiltonians}

In this section we present results for a variety of interaction strengths $0.4 \leqslant U \leqslant 10$ in the postquench Hamiltonian, as well as for quenches from the ground state at a finite value of the interactions. We provide two benchmarks for comparison.

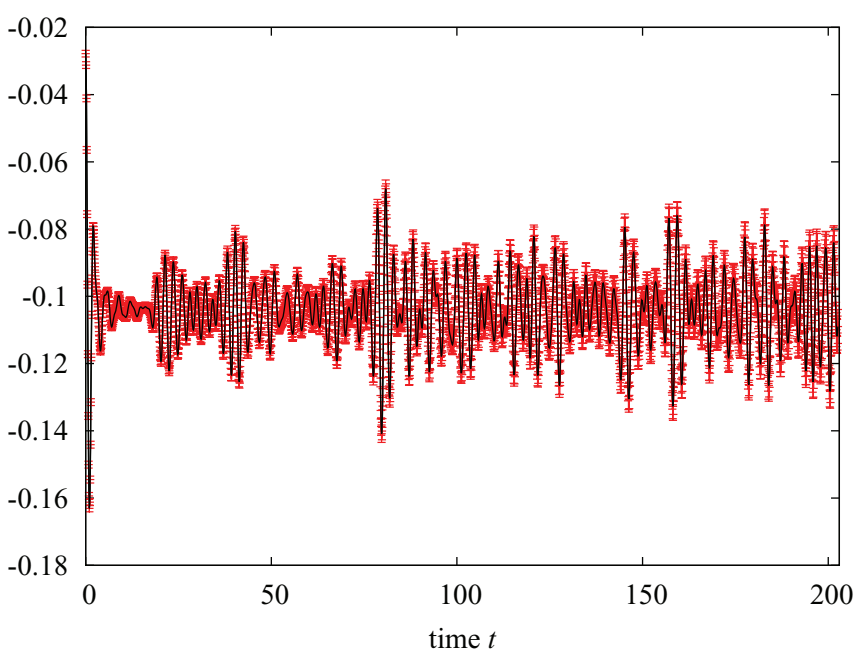

FIG. 21. (Color online) Time evolution of $\mathcal{G}(L / 2, L / 2+1)$ for quenches with $\delta_{i}=0.8 \rightarrow \delta_{f}=0.4$ and $U_{i}=0 \rightarrow U=0.4$. $L=$ 50 site system up to $t \sim 200$, with error bars estimated in Appendix B.
TABLE I. Summary of the effective temperature $\beta$ and chemical potential $\mu$ used in the QMC to calculate the Green's function $\mathcal{G}\left(\frac{L}{2}, \frac{L}{2}+1\right)$ on the $L=100$ chain as presented in Figs. 22-29. The energy density $E / L$ is found by taking the expectation value of the interacting Hamiltonian $H(t>0)$ at $t=0^{+}$.

\begin{tabular}{lccccc}
\hline \hline$U$ & $E / L$ & $\beta$ & $\mu$ & $\mathcal{G}\left(\frac{L}{2}, \frac{L}{2}+1\right)$ & $\begin{array}{c}\text { QMC } \\
\text { Error }\end{array}$ \\
\hline 0.4 & -0.664373 & 3.0741 & 0.4 & -0.46358 & $1.62 \times 10^{-3}$ \\
1 & -0.589142 & 2.6494 & 1 & -0.46247 & $2.98 \times 10^{-4}$ \\
2 & -0.463757 & 2.0437 & 2 & -0.44347 & $6.94 \times 10^{-5}$ \\
3 & -0.338371 & 1.5882 & 3 & -0.40153 & $6.49 \times 10^{-5}$ \\
4 & -0.212986 & 1.2175 & 4 & -0.34284 & $3.06 \times 10^{-4}$ \\
6 & 0.037784 & 0.7250 & 6 & -0.23885 & $1.34 \times 10^{-4}$ \\
8 & 0.288550 & 0.4868 & 8 & -0.17441 & $3.15 \times 10^{-4}$ \\
10 & 0.539324 & 0.3591 & 10 & -0.13514 & $1.23 \times 10^{-4}$ \\
\hline \hline
\end{tabular}

\section{Gibbs ensemble}

One useful comparison is with the appropriate Gibbs ensemble describing a putative thermal ensemble at late times. We have computed these by quantum Monte Carlo (QMC) using the ALPS collaboration [47] directed loop stochastic series expansion [48] code. Using the Jordan-Wigner transformation to map onto a spin model, the QMC calculations are performed in the grand canonical ensemble; the chemical potential and the effective temperature are fixed to ensure the correct energy and number densities (within the QMC error): these are given in Table I (see also Figs. 22-29). In the QMC simulations of the $L=100$ chain we perform $5 \times 10^{7}$ thermalization steps and perform measurements of the nearest-neighbor Green's function after $1.5 \times 10^{8}$ sweeps.

\section{Diagonal ensemble}

A second useful benchmark is provided by the diagonal ensemble. Given an initial state $\left|\Psi_{0}\right\rangle$ and a basis $\{|n\rangle\}$ of energy eigenstates, the diagonal ensemble average of an observable

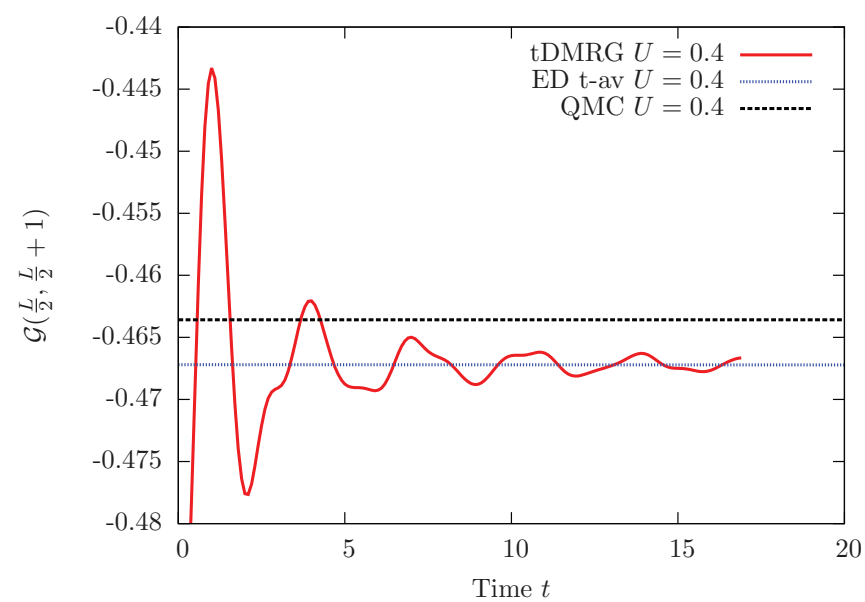

FIG. 22. (Color online) Comparison of the t-DMRG, timeaveraged (t-av) ED, and QMC results for the nearest-neighbor Green's function at time $t$ after the quench $\delta_{i}=0.8 \rightarrow \delta_{f}=0.4$, $U=0 \rightarrow 0.4$. t-DMRG and QMC simulations are performed on the $L=100$ chain, while ED studies the $L=16$ chain. 


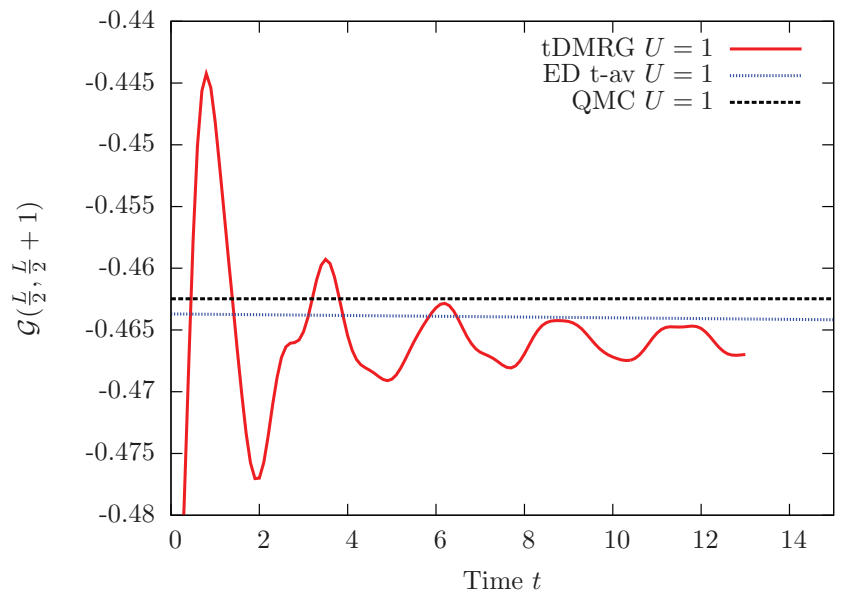

FIG. 23. (Color online) Comparison of the t-DMRG, timeaveraged (t-av) ED, and QMC results for the nearest-neighbor Green's function at time $t$ after the quench $\delta_{i}=0.8 \rightarrow \delta_{f}=0.4, U=0 \rightarrow 1$. t-DMRG and QMC simulations are performed on the $L=100$ chain, while ED studies the $L=16$ chain.

$\mathcal{O}$ is defined as

$$
\langle\mathcal{O}\rangle_{\mathrm{DE}}=\sum_{n}\langle n|\mathcal{O}| n\rangle\left|\left\langle n \mid \Psi_{0}\right\rangle\right|^{2} .
$$

For finite systems this equals the long-time average (over many recurrences). We compute the diagonal ensemble for a system of $L=16$ sites by exact diagonalization (ED).

\section{Difference between diagonal and Gibbs averages}

In Fig. 30 we show the difference between the expectations values of the nearest-neighbor Green's function $\mathcal{G}(L / 2, L / 2+$ 1 ) in the diagonal and Gibbs ensembles respectively for different values of $U_{f}$. As the diagonal ensemble is available

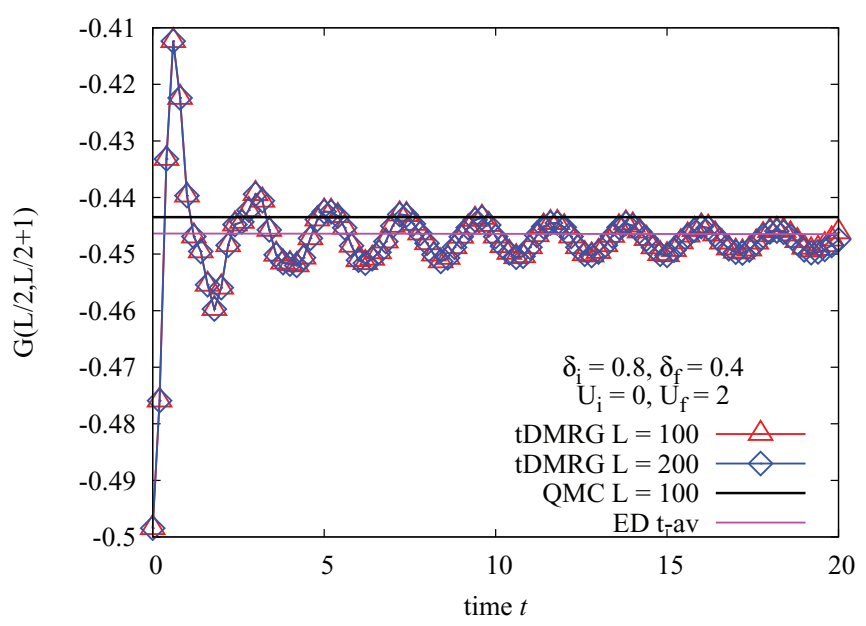

FIG. 24. (Color online) Comparison of the t-DMRG, timeaveraged (t-av) ED, and QMC results for the nearest-neighbor Green's function at time $t$ after the quench $\delta_{i}=0.8 \rightarrow \delta_{f}=0.4, U=0 \rightarrow 2$. t-DMRG simulations have been performed on the $L=100,200$ chain, QMC results are presented for the $L=100$ chain, and ED studies the $L=16$ chain.

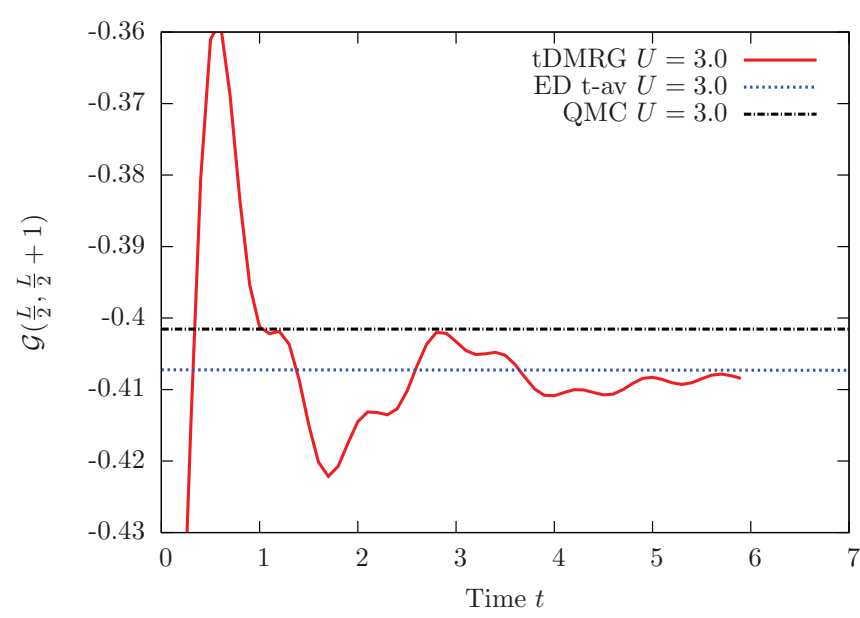

FIG. 25. (Color online) Comparison of the t-DMRG, timeaveraged (t-av) ED, and QMC results for the nearest-neighbor Green's function at time $t$ after the quench $\delta_{i}=0.8 \rightarrow \delta_{f}=0.4, U=0 \rightarrow 3$. t-DMRG and QMC simulations are performed on the $L=100$ chain, while ED studies the $L=16$ chain.

only for system size $L=16$, we display the quantities

$$
\begin{gathered}
\left\langle c_{L / 2}^{\dagger} c_{L / 2+1}\right\rangle_{\mathrm{DE}, \mathrm{L}=16}-\left\langle c_{L / 2}^{\dagger} c_{L / 2+1}\right\rangle_{\mathrm{Gibbs}, \mathrm{L}=16}, \\
\left\langle c_{L / 2}^{\dagger} c_{L / 2+1}\right\rangle_{\mathrm{DE}, \mathrm{L}=16}-\left\langle c_{L / 2}^{\dagger} c_{L / 2+1}\right\rangle_{\mathrm{Gibbs}, \mathrm{L}=100} .
\end{gathered}
$$

We see that for small values $U_{f}$ the two averages are close to one another, but for large $U_{f}$ they become very different.

\section{Results}

As can be seen from Figs. 22, 28, and 29, the nearestneighbor Green's function approaches plateau values at late times, which are compatible with the diagonal ensemble (given that the latter was calculated for $L=16$ we expect there to be finite-size effects), but not the Gibbs ensemble.

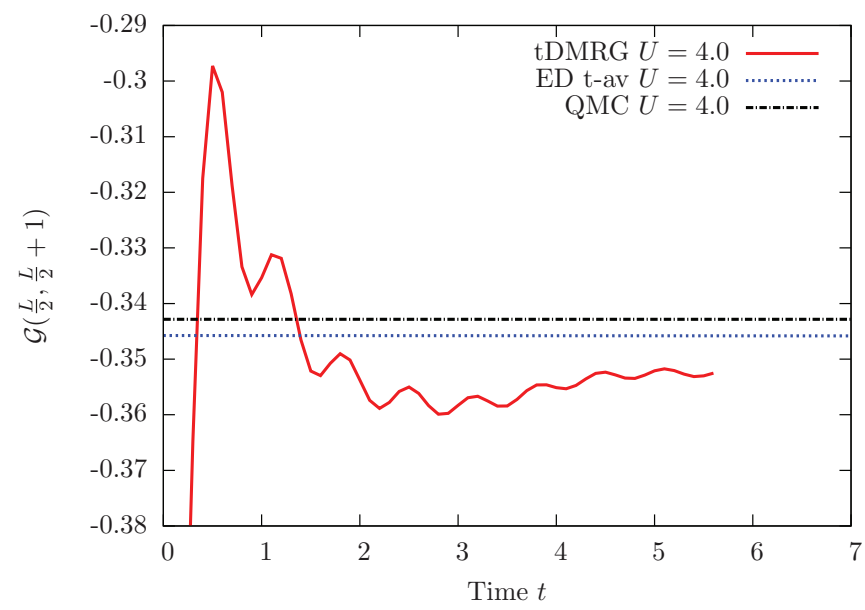

FIG. 26. (Color online) Comparison of the t-DMRG, timeaveraged (t-av) ED, and QMC results for the nearest-neighbor Green's function at time $t$ after the quench $\delta_{i}=0.8 \rightarrow \delta_{f}=0.4, U=0 \rightarrow 4$. t-DMRG and QMC simulations are performed on the $L=100$ chain, while ED studies the $L=16$ chain. 


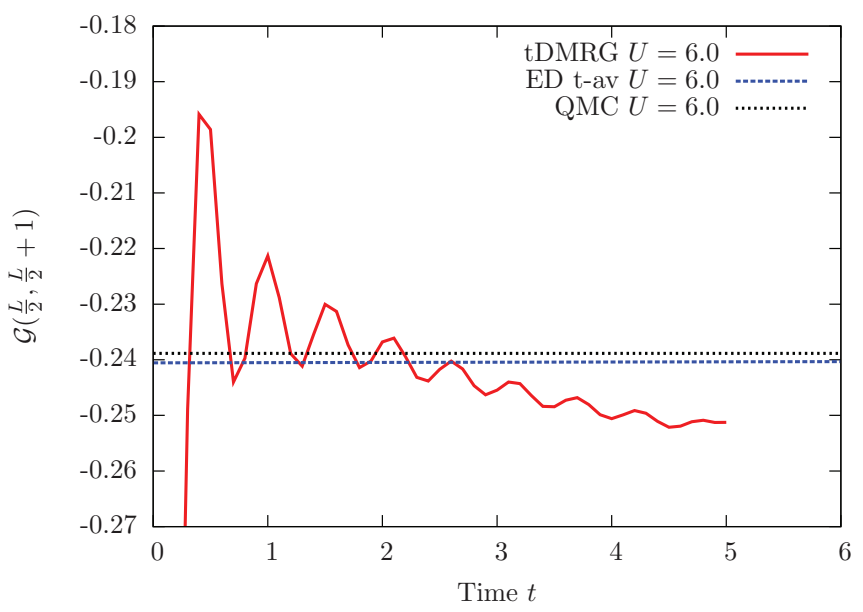

FIG. 27. (Color online) Comparison of the t-DMRG, timeaveraged (t-av) ED, and QMC results for the nearest-neighbor Green's function at time $t$ after the quench $\delta_{i}=0.8 \rightarrow \delta_{f}=0.4, U=0 \rightarrow 6$. t-DMRG and QMC simulations are performed on the $L=100$ chain, while ED studies the $L=16$ chain.

On the other hand, the plateau for intermediate values $U \approx$ 2 is compatible with a thermal ensemble on the accessible time scales. We propose the following explanation for these observations:

(1) The small- $U$ regime is described by a prethermalization plateau as discussed in Sec. V. It can be understood in terms of a deformation of the generalized Gibbs ensemble characterizing the stationary state of the $U=0$ quench.

(2) The large- $U$ regime is also described by a prethermalization plateau, which now can be understood in terms of a deformation of the generalized Gibbs ensemble characterizing the stationary state of the $\delta_{f}=0$ quench. This corresponds to a quench to the Heisenberg $X X Z$ chain in the massive regime. Given that our initial state has a short correlation length, GGE expectation values of local observables could be calculated by

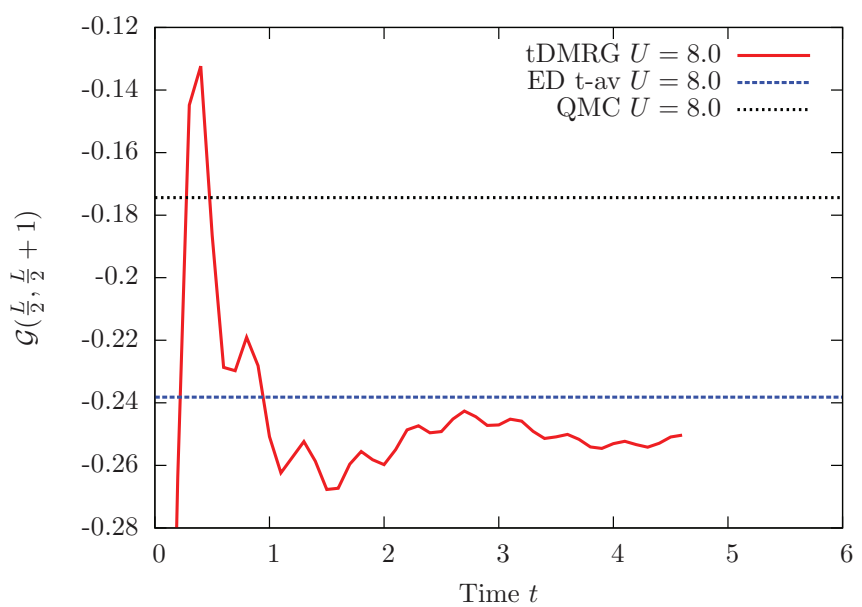

FIG. 28. (Color online) Comparison of the t-DMRG, timeaveraged (t-av) ED, and QMC results for the nearest-neighbor Green's function at time $t$ after the quench $\delta_{i}=0.8 \rightarrow \delta_{f}=0.4, U=0 \rightarrow 8$. t-DMRG and QMC simulations are performed on the $L=100$ chain, while ED studies the $L=16$ chain.

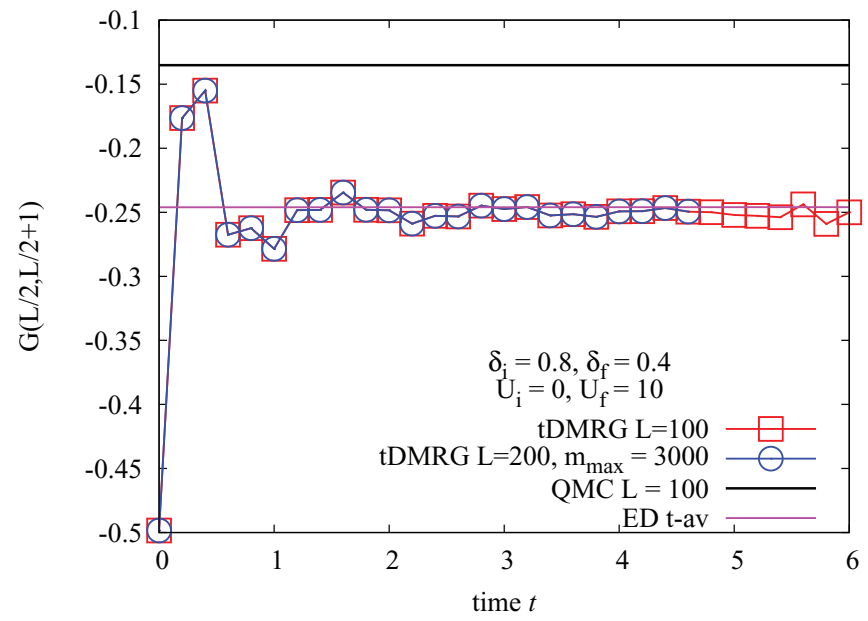

FIG. 29. (Color online) Comparison of the t-DMRG, timeaveraged (t-av) ED, and QMC results for the nearest-neighbor Green's function at time $t$ after the quench $\delta_{i}=0.8 \rightarrow \delta_{f}=0.4$, $U=0 \rightarrow 10$. t-DMRG simulations have been performed on the $L=100,200$ chain, QMC results are presented for the $L=100$ chain, and ED studies the $L=16$ chain.

the method of Ref. [24]. In order to test our interpretation, we have investigated the dependence of the plateau value on $\delta_{f}\left(\delta_{f}=0\right.$ corresponding to an integrable quench in the $X X Z$ chain). In Fig. 31 we show a comparison between quenches to $U_{f} \gg 1$ and $\delta_{f}=0$ or $\delta_{f}>0$, respectively. The correlator clearly approaches a plateau, the value of which is only very weakly dependent on the integrability-breaking parameter $\delta_{f}$, which supports our interpretation.

(3) In the intermediate- $U$ regime there is no prethermalization plateau, but the system relaxes slowly towards a Gibbs ensemble.

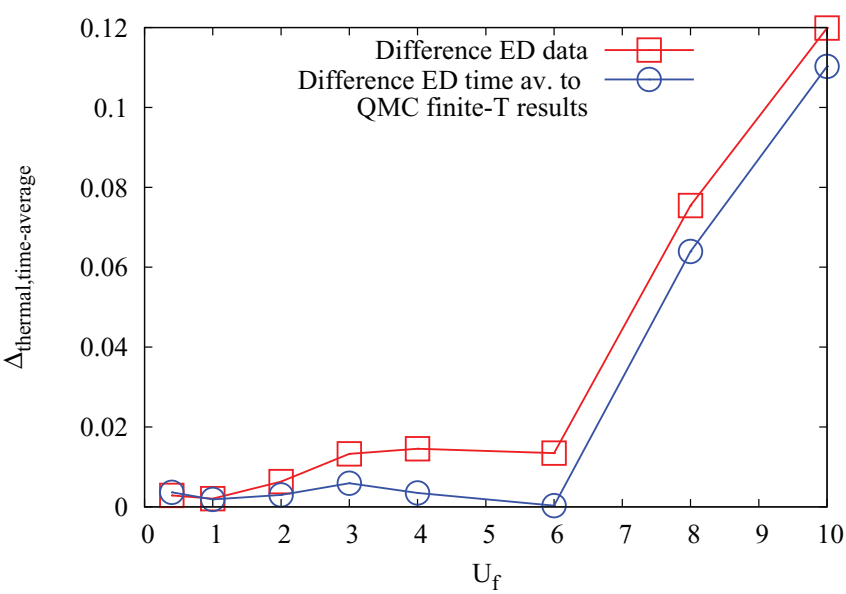

FIG. 30. (Color online) Difference in the value of $\mathcal{G}(L / 2, L / 2+$ 1) between finite-temperature results obtained with QMC $(L=100)$ or ED $(L=16)$ to the time-average values obtained via ED for $L=$ 16 for a quench with $\delta_{i}=0.8 \rightarrow \delta_{f}=0.4$ and $U_{i}=0 \rightarrow U_{f}$ as a function of $U_{f}$. Finite-size effects are less pronounced for small values of $U_{f}$, but prominent for $U_{f}>1$. The intermediate region $1 \leqslant U_{f}<8$ is the best candidate to obtain thermalization on long time scales in this system. 


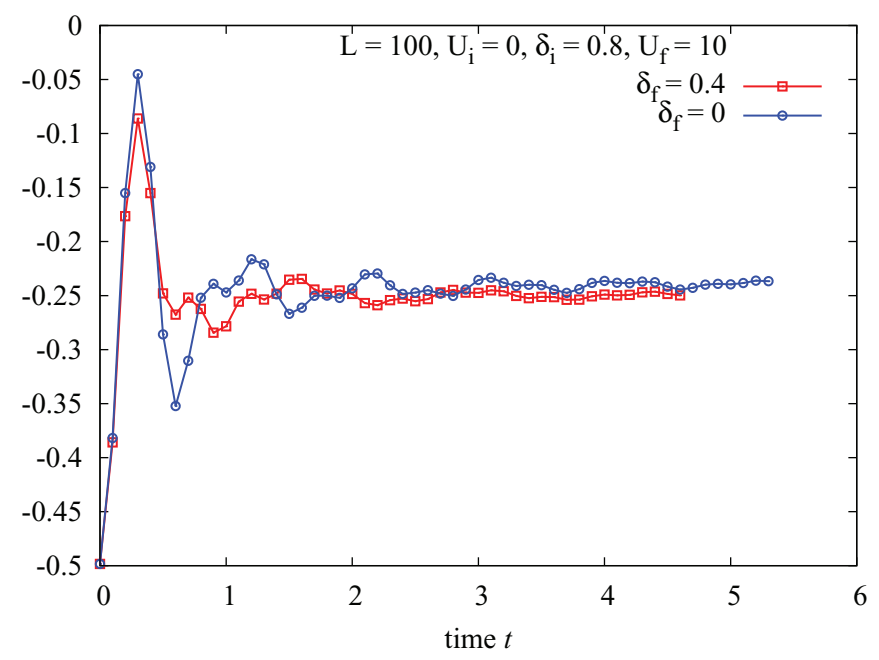

FIG. 31. (Color online) Comparison of t-DMRG results for the time evolution of $\mathcal{G}(L / 2, L / 2+1)$ for systems with $L=100$ sites for quenches with initial $U_{i}=0, \delta_{i}=0.8$ to values of $U_{f}=10$ and $\delta_{f}=0.4$ or $\delta_{f}=0$, respectively. As can be seen, the expectation value for both cases is very similar.

\section{Initial-state dependence}

A final issue we would like to address is whether our findings are sensitive to our particular choices of initial state. In order to assess this question we have carried out $\mathrm{t}$ DMRG computations for quenches starting in the ground state of strongly interacting Peierls insulators, i.e., Hamiltonians $H\left(\delta_{i}, U_{i}>0\right)$. Results for quenches of the form

$$
\left(\delta_{i}=0.8, U_{i}=5\right) \longrightarrow\left(\delta_{f}=0.4, U_{f}\right)
$$

with several values of $U_{f}$ are shown in Figs. 32 and 33. Here the expectation values of both the diagonal and Gibbs ensembles have been computed for $L=16$ site systems. Hence finite-size effects should be taken into account when making comparisons to the t-DMRG data.

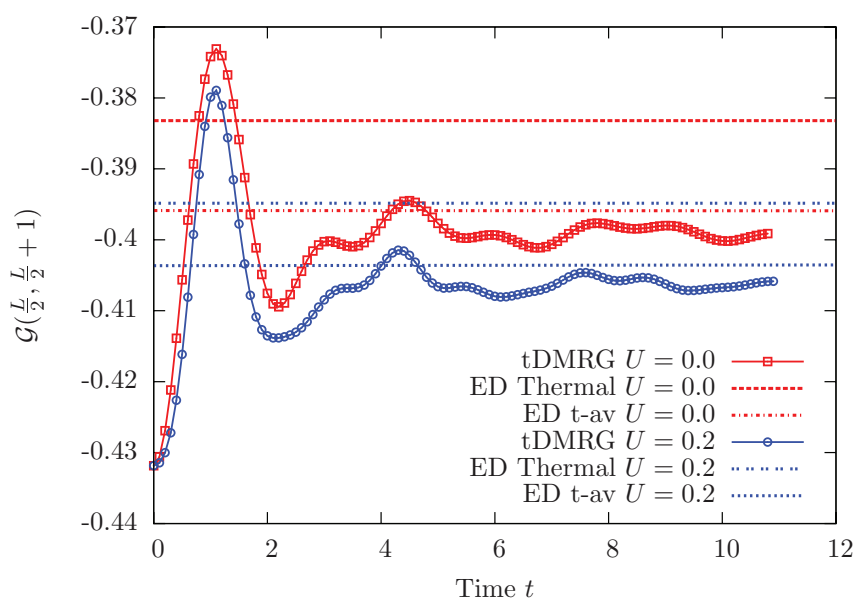

FIG. 32. (Color online) Green's function results from t-DMRG and ED for the quench $\delta_{i}=0.8 \rightarrow \delta_{f}=0.4$ with $U_{i}=5$ to $U=$ $0,0.2$. As with Figs. $22-29$ we see that the time-averaged (t-av) ED is compatible (up to finite-size effects) with the t-DMRG plateau value, while the thermal expectation is not.

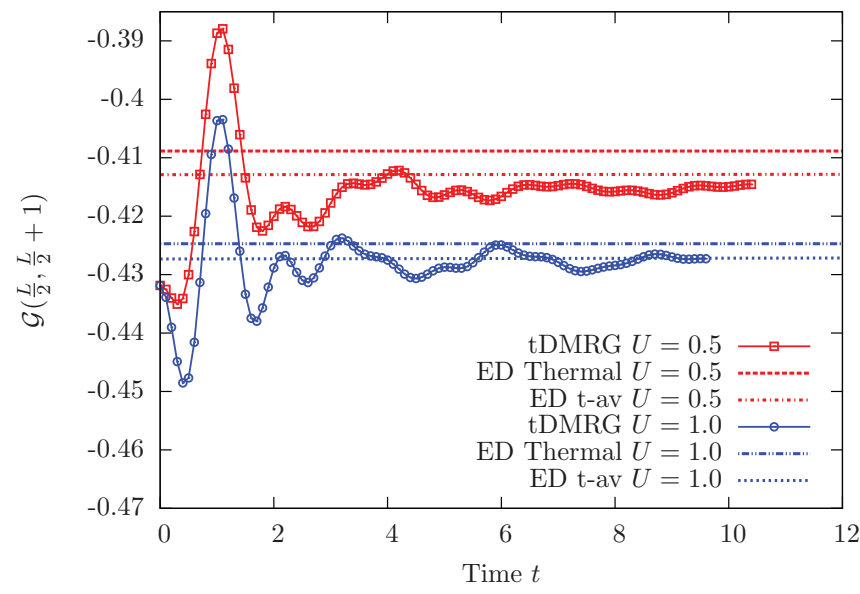

FIG. 33. (Color online) Green's function results from t-DMRG and ED for the quench $\delta_{i}=0.8 \rightarrow \delta_{f}=0.4$ with $U_{i}=5$ to $U=$ 0.5,1.0. As with Figs. $22-29$ we see that the time-averaged (t-av) ED is compatible (up to finite-size effects) with the t-DMRG plateau value.

The observed behavior is qualitatively very similar to that seen for quenches starting in noninteracting ground states. Observables relax to plateau values that are incompatible with thermalization when $U_{f}$ is either small or large.

\section{CONCLUSIONS}

Using a combination of analytical calculations based on the continuous unitary transform technique and time-dependent density matrix renormalization group computations we have established the existence of a robust prethermalization regime at intermediate times after a quantum quench to the weakly nonintegrable interacting Peierls insulator Hamiltonian (1). The combination of analytical and numerical techniques we use to analyze this plateau enables us to essentially eliminate finite-size effects. Our results thus represent true "bulk" physics, and in particular are free from revival effects. To the best of our knowledge, our work constitutes the first onedimensional example of a robust prethermalization plateau in a model with short-range interactions.

The CUT results allowed us to explicitly construct a "deformed generalized Gibbs ensemble," which provides an approximate statistical description of the prethermalization plateau. The deformed GGE is constructed from charges $\mathcal{Q}_{\alpha}(k)$, cf. Eq. (74), that form a mutually commuting set but do not commute with the Hamiltonian (44). As such, the deformed charges are not conserved at the operator level; only the expectation values $\left\langle\mathcal{Q}_{\alpha}(k)\right\rangle$ with respect to the time-evolved state $|\Psi(t)\rangle$ are approximately conserved. Our construction is therefore quite different from that of Ref. [33]. We conjecture that the deformed GGE idea applies more widely to quantum quenches in one-dimensional models with weak integrability breaking. It would be interesting to test this conjecture for other examples.

We expect that at very late times the system will actually thermalize, but we are not able to access sufficiently long times scales with either the perturbative CUT approach or t-DMRG. A possible approach to describe the dynamics at very late times 
might be through a quantum Boltzmann equation (see, e.g., Refs. [49]). This possibility is currently under investigation.

\section{ACKNOWLEDGMENTS}

We thank A. Chandran, M. Fagotti, M. Kolodrubetz, M. Rigol, and S. Sondhi for stimulating discussions. This work was supported by the EPSRC under Grants No. EP/I032487/1 (F.H.L.E. and N.J.R.) and No. EP/J014885/1 (F.H.L.E.). S.K. and S.R.M. acknowledge support through SFB 1073 of the Deutsche Forschungsgemeinschaft (DFG).

\section{APPENDIX A: LOCAL CONSERVATION LAWS FOR $\boldsymbol{H}_{0}$}

To derive the local conservation laws for the noninteracting Hamiltonian $H(\delta, 0)$ we follow Appendix C of Ref. [12]. Below we give the local conservation laws and summarize the salient points of the derivation.

The Hamiltonian can be written in the form

$$
H_{0}=\sum_{i, j=0}^{2 L-1} a_{i} \mathcal{H}_{i j} a_{j},
$$

where $a_{i}$ are Majorana fermions $\left\{a_{i}, a_{j}\right\}=2 \delta_{i, j}$ defined by

$$
\begin{gathered}
a_{2 n}=c_{n}^{\dagger}+c_{n}, \\
a_{2 n+1}=i\left(c_{n}^{\dagger}-c_{n}\right),
\end{gathered}
$$

and $\mathcal{H}$ is a skew-symmetric block-circulant matrix of the form

$$
\mathcal{H}=\left[\begin{array}{cccc}
\mathcal{Y}_{0} & \mathcal{Y}_{1} & \ldots & \mathcal{Y}_{\tilde{L}-1} \\
\mathcal{Y}_{\tilde{L}-1} & \mathcal{Y}_{0} & & \vdots \\
\vdots & & \ddots & \vdots \\
\mathcal{Y}_{1} & \ldots & \ldots & \mathcal{Y}_{0}
\end{array}\right]
$$

where $\mathcal{Y}_{n}$ are $4 \times 4$ matrices with $\mathcal{Y}_{n}=-\mathcal{Y}_{\tilde{L}-n}^{T}$ and $\tilde{L}=L / 2$. We define the Fourier transform of the block matrices as

$$
\left(\mathcal{Y}_{n}\right)_{j j^{\prime}}=\frac{1}{\tilde{L}} \sum_{k=1}^{\tilde{L}} e^{\frac{2 \pi i k}{\tilde{L}} n}\left(Y_{k}\right)_{j j^{\prime}}
$$

with $\left(Y_{k}\right)_{j n}=-\left(Y_{-k}\right)_{n j}$.

For free fermions a complete set of local conservation laws can be given by fermion bilinears

$$
I^{(r)}=\frac{1}{2} \sum_{l, n} a_{l} \mathcal{I}_{l n}^{(r)} a_{n},
$$

where the matrices $\mathcal{I}^{(r)}$ must satisfy

$$
\left[\mathcal{H}, \mathcal{I}^{(r)}\right]=0 \quad \text { and } \quad\left[\mathcal{I}^{(r)}, \mathcal{I}^{\left(r^{\prime}\right)}\right]=0 .
$$

The problem of deriving local conservation laws has now become the problem of finding a set of mutually commuting matrices that also commutes with the Hamiltonian matrix $\mathcal{H}$. At first sight the complexity of the problem does not seem to have been reduced, but we can now utilize a useful property of the Hamiltonian matrix $\mathcal{H}$ : the projectors onto eigenvectors of block circulant matrices are themselves block circulant matrices. This means one can consider $\mathcal{I}^{(r)}$ that are block circulant:

$$
\mathcal{I}^{(r)}=\left[\begin{array}{cccc}
\overline{\mathcal{Y}}_{0}^{(r)} & \overline{\mathcal{Y}}_{1}^{(r)} & \ldots & \overline{\mathcal{Y}}_{\tilde{L}-1}^{(r)} \\
\overline{\mathcal{Y}}_{\tilde{L}-1}^{(r)} & \overline{\mathcal{Y}}_{0}^{(r)} & & \vdots \\
\vdots & & \ddots & \vdots \\
\overline{\mathcal{Y}}_{1}^{(r)} & \ldots & \ldots & \overline{\mathcal{Y}}_{0}^{(r)}
\end{array}\right]
$$

Imposing Eqs. (A1), we obtain the conditions (for all $k$ )

$$
\left[Y_{k}, \bar{Y}_{k}^{(r)}\right]=0, \quad\left[\bar{Y}_{k}^{(r)}, \bar{Y}_{k}^{\left(r^{\prime}\right)}\right]=0,
$$

where $\bar{Y}_{k}^{(r)}$ is the Fourier transform of $\overline{\mathcal{Y}}^{(r)}$.

The construction of $\bar{Y}_{k}^{(r)}$ is straightforward as

$$
Y_{k}=A_{k} \otimes \sigma^{y}
$$

where

$$
\begin{aligned}
A_{k}= & {\left[J(1+\delta)+J(1-\delta) \cos \left(\frac{2 \pi k}{\tilde{L}}\right)\right] \sigma^{x} } \\
& -J(1-\delta) \sin \left(\frac{2 \pi k}{\tilde{L}}\right) \sigma^{y}
\end{aligned}
$$

So $\bar{Y}_{k}^{(r)}$ takes the form

$$
\begin{aligned}
\bar{Y}_{k}^{(r)}= & \bar{q}_{k}^{(r)} A_{k} \otimes \sigma^{y}+q_{k}^{(r)} A_{k} \otimes \mathbb{1}_{2} \\
& +\bar{\omega}_{k}^{(r)} \mathbb{1}_{2} \otimes \sigma^{y}+\omega_{k}^{(r)} \mathbb{1}_{2} \otimes \mathbb{1}_{2},
\end{aligned}
$$

where the functions $\omega_{k}^{(r)}, \bar{\omega}_{k}^{(r)}, q_{k}^{(r)}$, and $\bar{q}_{k}^{(r)}$ are chosen such that the Fourier transform satisfies $\left(\bar{Y}_{k}\right)_{j n}=-\left(\bar{Y}_{-k}\right)_{j n}$.

The ambiguity in choice of functions leads to different representations of the conservation laws; following Ref. [12] we make a particular choice that ensures there is a finite realspace range $r_{0}$ of the conservation laws: $\mathcal{I}_{l n}^{(r)}=0$ for $|l-n|>$ $r_{0}$. We consider the conservation laws associated with each of the terms in $\bar{Y}_{k}^{(r)}$ separately, and Fourier transforming back to real space we find

$$
\begin{aligned}
I_{1}^{(r)}= & -\sum_{n=0}^{\tilde{L}-1} \frac{J}{2}(1+\delta)\left[c_{2 n}^{\dagger} c_{2 n-2 r+3}+c_{2 n}^{\dagger} c_{2 n+2 r-1}+c_{2 n+1}^{\dagger} c_{2 n-2 r+2}+c_{2 n+1}^{\dagger} c_{2 n+2 r-2}+\text { H.c }\right] \\
& -\sum_{n=0}^{\tilde{L}-1} \frac{J}{2}(1-\delta)\left[c_{2 n}^{\dagger} c_{2 n-2 r+1}+c_{2 n}^{\dagger} c_{2 n+2 r-3}+c_{2 n+1}^{\dagger} c_{2 n-2 r+4}+c_{2 n+1}^{\dagger} c_{2 n+2 r}+\text { H.c. }\right],
\end{aligned}
$$



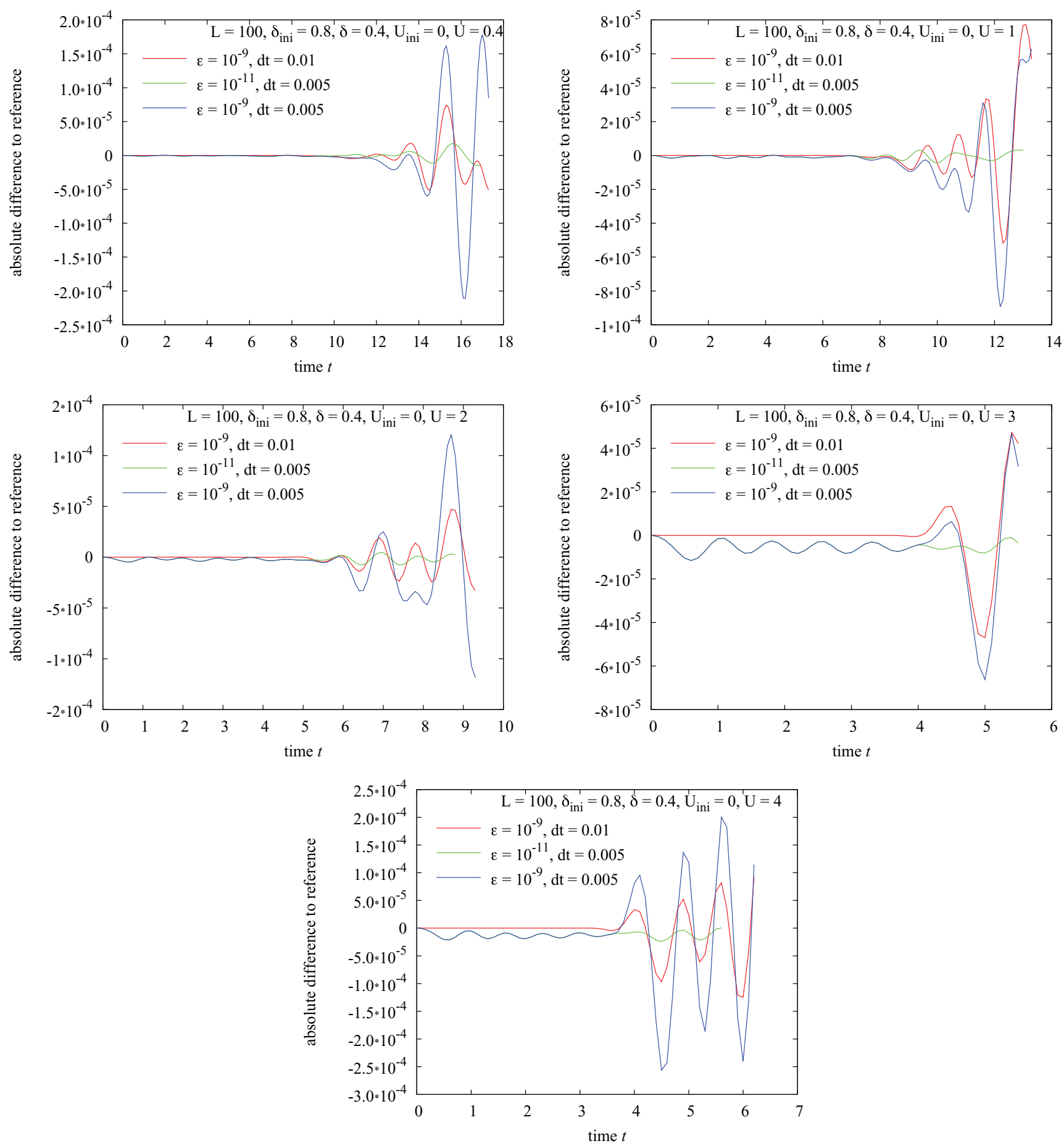

FIG. 34. (Color online) Differences between runs with different parameters and different quenches $(L=100$ in all cases).

$$
\begin{aligned}
I_{2}^{(r)}= & -\sum_{n=0}^{\tilde{L}-1} \frac{J}{2}(1+\delta)\left[i\left(c_{2 n}^{\dagger} c_{2 n-2 r+1}-c_{2 n}^{\dagger} c_{2 n+2 r+1}+c_{2 n+1}^{\dagger} c_{2 n-2 r}-c_{2 n+1}^{\dagger} c_{2 n+2 r}\right)+\text { H.c. }\right] \\
& -\sum_{n=0}^{\tilde{L}-1} \frac{J}{2}(1-\delta)\left[i\left(c_{2 n}^{\dagger} c_{2 n-2 r-1}-c_{2 n}^{\dagger} c_{2 n+2 r+1}+c_{2 n+1}^{\dagger} c_{2 n-2 r+2}-c_{2 n+1}^{\dagger} c_{2 n+2 r+2}\right)+\text { H.c. }\right], \\
I_{3}^{(r)}= & \sum_{n=0}^{\tilde{L}-1}\left[i\left(c_{2 n+2 r+2}^{\dagger} c_{2 n}+c_{2 n+1}^{\dagger} c_{2 n+2 r+3}\right)+\text { H.c. }\right], \quad I_{4}^{(r)}=\sum_{n=0}^{\tilde{L}-1}\left[i\left(c_{2 n+2 r+2}^{\dagger} c_{2 n}-c_{2 n+1}^{\dagger} c_{2 n+2 r+3}\right)+\text { H.c. }\right],
\end{aligned}
$$


where $r$ is a measure of the locality of the conservation laws and takes values 1 to $\tilde{L}$.

The local conservation laws $I_{3}^{(r)}, I_{4}^{(r)}$ are independent of the microscopic parameters of the theory; they arise from the $\mathbb{1}_{2} \otimes$ $\mathbb{1}_{2}$ and $\mathbb{1}_{2} \otimes \sigma^{y}$ terms in $\bar{Y}_{k}^{(r)}$. The remaining local conservation laws are dependent on the dimerization parameter $\delta$. Energy conservation is also manifest in the set of local conservation laws with $I_{1}^{(1)} \propto H_{0}$.

\section{APPENDIX B: ERROR ESTIMATE FOR THE t-DMRG}

In this appendix, we estimate the error for the long-time simulations. In principle, the error in a given observable can be estimated by the discarded weight $\varepsilon$, and due to the variational nature of the DMRG for ground-state calculations, it is $\sim \sqrt{\varepsilon}$ [50]. At short times this provides a reasonable estimate for time-evolved quantities as well. On longer time scales a number of complications emerge. (1) Due to the entanglement growth, the discarded weight grows quickly in time [51]. This can be addressed by adjusting the number of density matrix eigenstates, so that $\varepsilon$ is smaller than a chosen threshold (in our case $10^{-9}$ or $10^{-11}$ for some simulations).

(2) The error due to the Trotter decomposition becomes sizable. (3) Errors incurred in the sweeping procedure accumulate. In each DMRG step, the change of basis needed during the sweeps introduces an error $\sim \varepsilon$ as a result of the basis truncation. Hence, each sweep introduces an error $\sim L \varepsilon$ for a system of size $L$. This error is present at each time step. After a certain time $T$, a simulation with a step size $d t$ leads to an error $\sim(T / d t) L \varepsilon$. This error is in addition to the error in the observable due to the density matrix truncation discussed above. At short times the error due to the basis truncation $\sim \sqrt{\varepsilon}$ dominates, but at later times other error sources can no longer be neglected. This can be seen by varying both the target discarded weight and the time step. In Fig. 34 we show the difference of runs with different parameters to a reference run with $\varepsilon=10^{-11}$ and $d t=0.01$. The error between the results with a target discarded weight of $10^{-11}$ and $10^{-9}$ is seen to be roughly two orders of magnitudes,

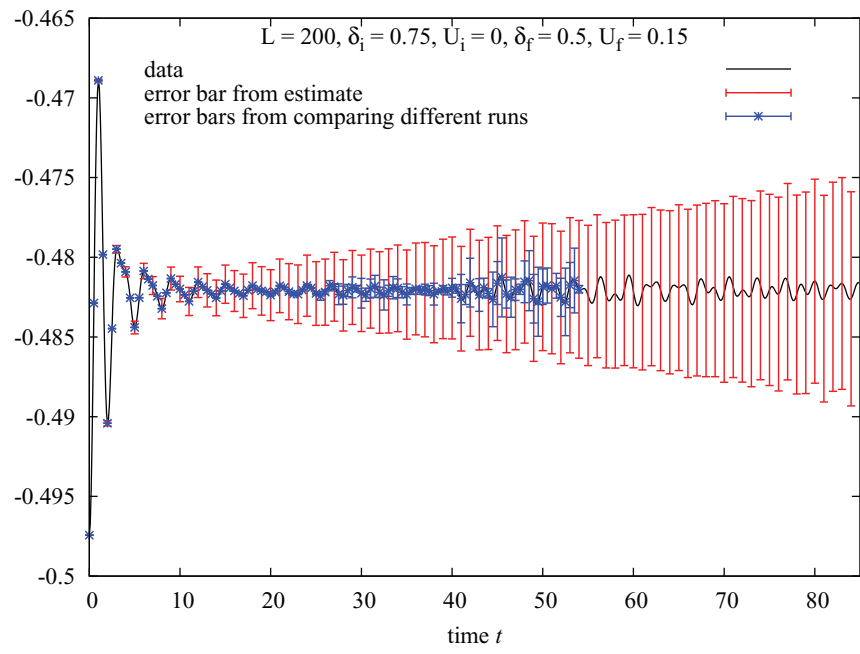

FIG. 35. (Color online) Error estimates for t-DMRG results on the time evolution of $\mathcal{G}(L / 2, L / 2+1)$ for a system with $L=200$ sites and a quench $\delta_{i}=0.75 \rightarrow \delta_{f}=0.5$ and $U_{i}=0 \rightarrow U_{f}=0.5$. The data are obtained using a time step of $\delta t=0.005$ and a target discarded weight of $\varepsilon=10^{-9}$. The red error bars (lines) are obtained from the estimate discussed in Appendix B; the blue ones (asterisks) are obtained by comparing to the results of a run with time step $\delta t=0.01$. The error estimate appears to be larger, but of similar order of magnitude to the actual deviation between the results at times $\sim 50$. From this estimate we obtain at the end of the time evolution a relative error of the order of $1.5 \%$.

as expected from the above estimate. The error bars shown in Figs. 21 and 35 are estimated on the basis of the above considerations. The error bars grow significantly towards the end of the time evolution, but still permit us to make qualitative statements. For the runs considered, this indicates that on the time scales treated the quasistationary state does not change; i.e., the prethermalization plateau is still present. Together with ED results obtained for small systems for times up to $t=1000$, this indicates that thermalization happens at much larger time scales $(\gg 100)$, if at all.
[1] M. Greiner, O. Mandel, T. W. Hänsch, and I. Bloch, Nature (London) 419, 51 (2002).

[2] T. Kinoshita, T. Wenger, and D. S. Weiss, Nature (London) 440, 900 (2006); http://jila.colorado.edu/ USJAPAN/pdf/Kinoshita.pdf.

[3] S. Hoerberth, I. Lesanovsky, B. Fischer, T. Schumm, and J. Schmiedmayer, Nature (London) 449, 324 (2007).

[4] S. Trotzky, Y.-A. Chen, A. Flesch, I. P. McCulloch, U. Schöllwock, J. Eisert, and I. Bloch, Nat. Phys. 8, 325 (2012).

[5] M. Cheneau, P. Barmettler, D. Poletti, M. Endres, P. Schauss, T. Fukuhara, C. Gross, I. Bloch, C. Kollath, and S. Kuhr, Nature (London) 481, 484 (2012).

[6] M. Gring, M. Kuhnert, T. Langen, T. Kitagawa, B. Rauer, M. Schreitl, I. Mazets, D. A. Smith, E. Demler, and J. Schmiedmayer, Science 337, 1318 (2012).
[7] A. Polkovnikov, K. Sengupta, A. Silva, and M. Vengalattore, Rev. Mod. Phys. 83, 863 (2011).

[8] J. M. Deutsch, Phys. Rev. A 43, 2046 (1991); M. Srednicki, Phys. Rev. E 50, 888 (1994); M. Rigol, V. Dunjko, and M. Olshanii, Nature (London) 452, 854 (2008); E. Canovi, D. Rossini, R. Fazio, G. Santoro, and A. Silva, New J. Phys. 14, 095020 (2012).

[9] T. Barthel and U. Schollwöck, Phys. Rev. Lett. 100, 100601 (2008).

[10] M. Cramer, C. M. Dawson, J. Eisert, and T. J. Osborne, Phys. Rev. Lett. 100, 030602 (2008); M. Cramer and J. Eisert, New J. Phys. 12, 055020 (2010).

[11] P. Calabrese, F. H. L. Essler, and M. Fagotti, Phys. Rev. Lett. 106, 227203 (2011); J. Stat. Mech.: Theory Exp. (2012) P07016; (2012) P07022. 
[12] M. Fagotti and F. H. L. Essler, Phys. Rev. B 87, 245107 (2013).

[13] P. Calabrese and J. Cardy, J. Stat. Mech.: Theory Exp. (2007) P06008.

[14] A. Iucci and M. A. Cazalilla, Phys. Rev. A 80, 063619 (2009).

[15] G. Biroli, C. Kollath, and A. M. Läuchli, Phys. Rev. Lett. 105, 250401 (2010).

[16] D. Fioretto and G. Mussardo, New J. Phys. 12, 055015 (2010).

[17] F. H. L. Essler, S. Evangelisti, and M. Fagotti, Phys. Rev. Lett. 109, 247206 (2012).

[18] B. Pozsgay, J. Stat. Mech.: Theory Exp. (2011) P01011.

[19] A. C. Cassidy, C. W. Clark, and M. Rigol, Phys. Rev. Lett. 106, 140405 (2011).

[20] M. A. Cazalilla, A. Iucci, and M.-C. Chung, Phys. Rev. E 85, 011133 (2012).

[21] J.-S. Caux and R. M. Konik, Phys. Rev. Lett. 109, 175301 (2012).

[22] J. Mossel and J.-S. Caux, New J. Phys. 14, 075006 (2012).

[23] J.-S. Caux and F. H. L. Essler, Phys. Rev. Lett. 110, 257203 (2013).

[24] M. Fagotti and F. H. L. Essler, J. Stat. Mech.: Theory Exp. (2013) P07012.

[25] B. Pozsgay, J. Stat. Mech.: Theory Exp. (2013) P07003.

[26] G. Mussardo, arXiv:1304.7599.

[27] M. Collura, S. Sotiriadis, and P. Calabrese, Phys. Rev. Lett. 110, 245301 (2013); J. Stat. Mech.: Theory Exp. (2013) P09025.

[28] M. Kormos, M. Collura, and P. Calabrese, Phys. Rev. A 89, 013609 (2014).

[29] M. Kormos, A. Shashi, Y.-Z. Chou, J.-S. Caux, and A. Imambekov, Phys. Rev. B 88, 205131 (2013).

[30] M. Rigol, V. Dunjko, V. Yurovsky, and M. Olshanii, Phys. Rev. Lett. 98, 050405 (2007).

[31] S. R. Manmana, S. Wessel, R. M. Noack, and A. Muramatsu, Phys. Rev. Lett. 98, 210405 (2007); Phys. Rev. B 79, 155104 (2009); C. Kollath, A. M. Läuchli, and E. Altman, Phys. Rev. Lett. 98, 180601 (2007).

[32] M. Moeckel and S. Kehrein, Phys. Rev. Lett. 100, 175702 (2008); Ann. Phys. 324, 2146 (2009); New J. Phys. 12, 055016 (2010).

[33] M. Kollar, F. A. Wolf, and M. Eckstein, Phys. Rev. B 84, 054304 (2011).
[34] M. Rigol, Phys. Rev. Lett. 103, 100403 (2009); Phys. Rev. A 80, 053607 (2009); L. F. Santos and M. Rigol, Phys. Rev. E 81, 036206 (2010); 82, 031130 (2010).

[35] M. Marcuzzi, J. Marino, A. Gambassi, and A. Silva, Phys. Rev. Lett. 111, 197203 (2013).

[36] G. Brandino, J.-S. Caux, and R. M. Konik, arXiv:1301.0308.

[37] T. Kitagawa, A. Imambekov, J. Schmiedmayer, and E. Demler, New J. Phys. 13, 073018 (2011).

[38] T. Langen, M. Gring, M. Kuhnert, B. Rauer, R. Geiger, D. A. Smith, I. E. Mazets, and J. Schmiedmayer, Eur. Phys. J. Special Topics 217, 43 (2013).

[39] V. Y. Krivnov and A. A. Ovchinnikov, Zh. Eksp. Teor. Fiz. 90, 709 (1986) [Sov. Phys. JETP 63, 414 (1986)].

[40] C. Schuster and U. Eckern, Eur. Phys. J. B 5, 395 (1998).

[41] T. Nakano and H. Fukuyama, J. Phys. Soc. Jpn 50, 2489 (1981); F. H. L. Essler and R. M. Konik, in From Fields to Strings: Circumnavigating Theoretical Physics, edited by M. Shifman, A. Vainshtein, and J. Wheater (World Scientific, Singapore, 2005); arXiv:cond-mat/0412421.

[42] In practice we consider a very large system of size $L$ and take into account $L$ conserved quantities.

[43] J. Sirker, N. P. Konstantinidis, F. Andraschko, and N. Sedlmayr, arXiv:1303.3064.

[44] F. Wegner, Ann. Phys. 506, 77 (1994); J. Phys. A 39, 8221 (2006); S. D. Glazek and K. G. Wilson, Phys. Rev. D 48, 5863 (1993); 49, 4214 (1994).

[45] S. Kehrein, The Flow Equation Approach to Many-Particle Systems (Springer, Berlin, 2006)

[46] S. Kehrein, Phys. Rev. Lett. 95, 056602 (2005); A. Hackl and S. Kehrein, Phys. Rev. B 78, 092303 (2008).

[47] B. Bauer et al. (ALPS Collaboration), J. Stat. Mech.: Theory Exp. (2011) P05001.

[48] A. W. Sandvik and J. Kurkijärvi, Phys. Rev. B 43, 5950 (1991); A. W. Sandvik, J. Phys. A 25, 3667 (1992).

[49] L. P. Kadanoff and G. Baym, Quantum Statistical Mechanics (Benjamin, New York, 1962); J. Rammer and H. Smith, Rev. Mod. Phys. 58, 323 (1986); M. L. R. Fürst, C. B. Mendl, and H. Spohn, Phys. Rev. E 86, 031122 (2012); M. Tavora and A. Mitra, Phys. Rev. B 88, 115144 (2013).

[50] S. R. White and A. L. Chernyshev, Phys. Rev. Lett. 99, 127004 (2007).

[51] D. Gobert, C. Kollath, U. Schollwöck, and G. Schütz, Phys. Rev. E 71, 036102 (2005). 